\title{
EQUILIBRIUM STATES OF WEAKLY HYPERBOLIC ONE-DIMENSIONAL MAPS FOR HÖLDER POTENTIALS
}

\author{
HUAIBIN LI AND JUAN RIVERA-LETELIER
}

\begin{abstract}
There is a wealth of results in the literature on the thermodynamic formalism for potentials that are, in some sense, "hyperbolic". We show that for a sufficiently regular one-dimensional map satisfying a weak hyperbolicity assumption, every Hölder continuous potential is hyperbolic. A sample consequence is the absence of phase transitions: The pressure function is real analytic on the space of Hölder continuous functions. Another consequence is that every Hölder continuous potential has a unique equilibrium state, and that this measure has exponential decay of correlations.
\end{abstract}

\section{INTRODUCTION}

The thermodynamic formalism of smooth dynamical systems was initiated by Sinai, Ruelle, and Bowen [Bow75, Rue76, Sin72]. For a uniformly hyperbolic diffeomorphism acting on a compact manifold of arbitrary dimension, they gave a complete description for Hölder continuous potentials. In particular, they showed that on each basic set there is a unique equilibrium state, and that this measure has exponential decay of correlations. Furthermore, there are no phase transitions: The topological pressure of a basic set is real analytic as a function of the potential.

There have been several extensions of these results to one-dimensional maps, that go beyond the uniformly hyperbolic setting. The lack of uniform hyperbolicity is usually compensated by an extra hypothesis on the potential. For example, there is a wealth of results for a piecewise monotone interval map $f: I \rightarrow I$ and a potential $\varphi$ of bounded variation satisfying

$$
\sup _{I} \varphi<P(f, \varphi)
$$

where $P(f, \varphi)$ denotes the pressure. Most results apply under the following weaker condition:

For some integer $n \geq 1$, the function $S_{n}(\varphi):=\sum_{j=0}^{n-1} \varphi \circ f^{j}$ satisfies

$$
\sup _{I} \frac{1}{n} S_{n}(\varphi)<P(f, \varphi) \text {. }
$$

2010 Mathematics Subject Classification. Primary 37D35; Secondary 37E05, 37F10.

Key words and phrases. One-dimensional dynamical systems, non-uniform hyperbolicity, thermodynamic formalism.

HL was partially supported by the National Natural Science Foundation of China (Grant No. 11101124) and FONDECYT grant 3110060 of Chile.

JRL was partially supported by FONDECYT grant 1100922 of Chile. 
In what follows, a potential $\varphi$ satisfying this condition is said to be hyperbolic for $f$. See for example [BK90, DKU90, HK82, Kel85, LSV98, Rue94 and references therein, as well as Baladi's book [Bal00, §3]. The classical result of Lasota and Yorke [LY73] can be recovered as the special case in which $f$ is piecewise $C^{2}$ and uniformly expanding, and $\varphi=-\log |D f|$.

For a complex rational map in one variable $f$, and a Hölder continuous potential $\varphi$ that is hyperbolic for $f$, a complete description of the thermodynamic formalism was given by Denker, Haydn, Przytycki, and Urbański in Hay99, DPU96, DU91, Prz90, * extending previous results of Freire, Lopes, and Mañé [FLM83, Mañ83, and Ljubich Lju83. See also the alternative approach of Szostakiewicz, Urbański, and Zdunik in [SUZ11, and [FU00, Hay00, IRRL12] for further results.

Using the results of Keller in [Kel85], we recently extended these results to the case of a sufficiently regular interval map and a Hölder continuous potential in the companion paper [LRL13]. See [BT08] for an earlier result in a similar setting.

Suitably interpreted, the hyperbolicity condition also appears in the study of geometric potentials in PRL11, PRL13]. In fact, consider a continuous map $f$ acting on a compact metric space $X$, and let $\mathcal{M}(X, f)$ be the space of Borel probability measures on $X$ that are invariant by $f$. Then a continuous potential $\varphi$ is hyperbolic if and only if

$$
\sup _{\mu \in \mathcal{M}(X, f)} \int_{X} \varphi d \mu<P(f, \varphi)
$$

see for example [IRRL12, Proposition 3.1]. In the case $f$ is a differentiable map in one real or complex variable, put

$$
\chi_{\mathrm{inf}}:=\inf _{\mu \in \mathcal{M}(X, f)} \int_{X} \log |D f| d \mu \text { and } \chi_{\mathrm{sup}}:=\sup _{\mu \in \mathcal{M}(X, f)} \int_{X} \log |D f| d \mu .
$$

Then for each real number $t$, the condition (1.1) applied to the potential $\varphi=$ $-t \log |D f|$ becomes

$$
P(f,-t \log |D f|)>\max \left\{-t \chi_{\mathrm{inf}},-t \chi_{\mathrm{sup}}\right\},
$$

which is precisely the condition appearing in [PRL11, PRL13].

In this paper we show, in both the real and the complex setting, that for a sufficiently regular one-dimensional map satisfying a weak form of hyperbolicity, every Hölder continuous potential is hyperbolic. So, for such a map $f$, all the results above apply to every Hölder continuous potential. A consequence is that for every Hölder continuous function $\varphi$ there is a unique equilibrium state of $f$ for the potential $\varphi$. Moreover, this measure has several statistical properties, like exponential decay of correlations and the Almost Sure Invariance Principle, see Remark 1.1. Another consequence

\footnotetext{
${ }^{*}$ In this setting most of the results have been stated for a potential $\varphi$ satisfying the condition $\sup \varphi<P(f, \varphi)$ that is more restrictive than $\varphi$ being hyperbolic for $f$. However, general arguments show they also apply to hyperbolic potentials, see [RRL12, §3].
} 
is the absence of phase transitions: The pressure function is real analytic on the space of Hölder continuous functions. In [Li13] this result is used to show a level-2 Large Deviation Principle for Hölder continuous potentials.

We proceed to describe our main results more precisely.

1.1. Statements of results. We state 2 results, one in the real setting and the other in the complex setting. To simplify the exposition, they are formulated in a more restricted situation than what we are able to handle, see the Main Theorem in $₫ 2$ for a more general formulation of our results. We start recalling some concepts from thermodynamic formalism, see for example [PU10, Wal78] for background.

Let $(X$, dist) be a compact metric space and $T: X \rightarrow X$ a continuous map. Denote by $\mathcal{M}(X)$ the space of Borel probability measures on $X$ endowed with the weak* topology, and by $\mathcal{M}(X, T)$ the subspace of $\mathcal{M}(X)$ of those measures that are invariant by $T$. For each measure $\nu$ in $\mathcal{M}(X, T)$, denote by $h_{\nu}(T)$ the measure-theoretic entropy of $\nu$. For a continuous function $\varphi: X \rightarrow \mathbb{R}$, denote by $P(T, \varphi)$ the topological pressure of $T$ for the potential $\varphi$, defined by

$$
P(T, \varphi):=\sup \left\{h_{\nu}(T)+\int_{X} \varphi d \nu: \nu \in \mathcal{M}(X, T)\right\} .
$$

A measure $\nu$ in $\mathcal{M}(X, T)$ is called an equilibrium state of $T$ for the potential $\varphi$, if the supremum in (1.2) is attained at $\nu$.

On the other hand, a measure $\nu$ in $\mathcal{M}(X, T)$ is exponentially mixing for $f$ or has exponential decay of correlations for $f$, if there are constants $C>0$ and $\rho$ in $(0,1)$, such that for every bounded measurable function $\phi: X \rightarrow \mathbb{R}$ and every Lipschitz continuous function $\psi: X \rightarrow \mathbb{R}$, we have for every integer $n \geq 1$

$$
\left|\int_{X} \phi \circ f^{n} \cdot \psi d \nu-\int_{X} \phi d \nu \int_{X} \psi d \nu\right| \leq C\left(\sup _{X}|\phi|\right)\|\psi\|_{\text {Lip }} \cdot \rho^{n},
$$

where $\|\psi\|_{\text {Lip }}:=\sup _{x, x^{\prime} \in X, x \neq x^{\prime}} \frac{\left|\psi(x)-\psi\left(x^{\prime}\right)\right|}{\operatorname{dist}\left(x, x^{\prime}\right)}$.

Given a compact interval $I$, a smooth map $f: I \rightarrow I$ is non-degenerate, if the number of points at which the derivative of $f$ vanishes is finite, and if for every such point there is a higher order derivative of $f$ that is non-zero.

Theorem A. Let I be a compact interval and let $f: I \rightarrow I$ be a topologically exact non-degenerate smooth map. Assume $f$ has only hyperbolic repelling periodic points, and for each critical value $v$ of $f$ we have

$$
\lim _{n \rightarrow+\infty}\left|D f^{n}(v)\right|=+\infty .
$$

Then every Hölder continuous potential $\varphi: I \rightarrow \mathbb{R}$ is hyperbolic for $f$. In particular, there is a unique equilibrium state $\nu$ of $f$ for the potential $\varphi$. Moreover, the measure-theoretic entropy of $\nu$ is positive, and $\nu$ is exponentially mixing for $f$. Finally, for every Hölder function $\psi: I \rightarrow \mathbb{R}$, the function $t \mapsto P(f, \varphi+t \psi)$ is real analytic. 
Under the additional assumption that $\varphi$ is hyperbolic for $f$, the assertions of Theorem $\mathrm{A}$ can be obtained by combining [BPS01, Corollaries 1.1 and 1.2] and [RLS10, Theorem A]. Under the more restrictive bounded range condition: $\sup _{I} \varphi-\inf _{I} \varphi<h_{\text {top }}(f)$, these are given by [Kel85, Theorem 3.4], see also [BT08, BT11. Theorem A shows that the assumption that $\varphi$ is hyperbolic for $f$ is not needed.

Buzzi showed a result related to Theorem A for potentials that are "symbolically Hölder continuous", see [Buz04, Theorem 1]. For a smooth nondegenerate map $f$, the class of symbolically Hölder continuous potentials is not related to the class of Hölder continuous potentials, unless $f$ satisfies the Topological Collet-Eckmann condition, see [RL12a, Corollary C]. If $f$ satisfies the Topological Collet-Eckmann condition, then these classes of potentials coincide and our argument gives a different proof of Buzzi's result When $f$ has only one critical value $v$, the Topological Collet-Eckmann condition is equivalent to the condition that $\left|D f^{n}(v)\right|$ grows exponentially in $n$, so our result is significantly stronger in this case.

Once it is shown that the potential $\varphi$ is hyperbolic for $f$, the rest of the assertions of Theorem A follow from LRL13, Corollary 1.4].

In contrast with Theorem A, our Main Theorem in $\$ 2$ applies to maps having a neutral periodic point. The prototypical examples of interval maps having a neutral periodic point are the intermittent maps studied in LSV99, Lop93, MT12, PM80, PS92, PSY98, PW99, Sar01, YYu99, among others. For concreteness, fix $\alpha$ in $(0,1)$ and consider the map

$$
\begin{aligned}
f_{\alpha}:[0,1] & \rightarrow[0,1] \\
x & \mapsto \begin{cases}x\left(1+x^{\alpha}\right) & \text { if } x\left(1+x^{\alpha}\right) \leq 1 ; \\
x\left(1+x^{\alpha}\right)-1 & \text { otherwise. }\end{cases}
\end{aligned}
$$

Our argument shows that for each $\alpha^{\prime}$ in $(\alpha, 1]$, every Hölder continuous potential of exponent $\alpha^{\prime}$ is hyperbolic for $f_{\alpha}$. In particular, $f_{\alpha}$ has no phase transitions on the space of Hölder continuous potentials of exponent $\alpha^{\prime}$. The hypothesis that $\alpha^{\prime}$ is in $(\alpha, 1]$ is necessary: The potential $-\log \left|D f_{\alpha}\right|$ is Hölder continuous of exponent $\alpha$, and it is not hyperbolic for $f_{\alpha}$, see Remark 2.4.

To state our main result in the complex setting, for each complex rational map $f$ denote by Crit $(f)$ the set of critical points of $f$, and by $J(f)$ the Julia set of $f$. In what follows we restrict the action of $f$ to $J(f)$. In particular, the pressure function is defined through measures supported on $J(f)$ and each equilibrium state is supported on $J(f)$.

Theorem B. Let $f$ be one of the following:

1. An at most finitely renormalizable complex polynomial of degree at least 2, without neutral cycles and such that for each critical value $v$

\footnotetext{
${ }^{\dagger}$ Although there are maps satisfying the Topological Collet-Eckmann condition that do not satisfy the hypotheses of Theorem A our Main Theorem in $\$ 2$ does apply to maps satisfying the Topological Collet-Eckmann condition, see Remark 2.3
} 
of $f$ we have

$$
\lim _{n \rightarrow+\infty}\left|D f^{n}(v)\right|=+\infty
$$

2. A complex rational map of degree at least 2 , without parabolic cycles and such that for every critical value $v$ of $f$ we have

$$
\sum_{n=1}^{+\infty} \frac{1}{\left|D f^{n}(v)\right|}<+\infty .
$$

Then every Hölder continuous potential $\varphi: J(f) \rightarrow \mathbb{R}$ is hyperbolic for $f$. In particular, there is a unique equilibrium state $\nu$ of $f$ for the potential $\varphi$. Moreover, the measure-theoretic entropy of $\nu$ is positive and $\nu$ is exponentially mixing for $f$. Finally, for every Hölder function $\psi: I \rightarrow \mathbb{R}$, the function $t \mapsto P(f, \varphi+t \psi)$ is real analytic.

Recall that for an integer $s \geq 2$, a complex polynomial $f$ is renormalizable of period $s$, if there are Jordan disks $U$ and $V$ in $\mathbb{C}$, such that $\bar{U} \subset V$ and such that the following hold:

- $f^{s}: U \rightarrow V$ is proper of degree at least 2 ;

- The set $\left\{z \in U: f^{s n}(z) \in U\right.$ for all $\left.n=1,2, \ldots\right\}$ is connected and strictly contained in $J(f)$;

- For each critical point $c$ of $f$ there exists at most one $j$ in $\{0,1, \ldots, s-$ 1 ) such that $c$ is $f^{j}(U)$.

We say that $f$ is infinitely renormalizable if there are infinitely many integers $s \geq 2$ such that $f$ is renormalizable of period $s$.

In the case where $f$ is a complex rational map satisfying the Topological Collet-Eckmann condition, the essential part of Theorem B is IRRL12, Corollary 1.2]. We note that the results of [RRL12 only apply to maps having no invariant measure of zero Lyapunov exponent, so Theorem $B$ is substantially stronger.

Once it is shown that the potential $\varphi$ is hyperbolic for $f$, the rest of the assertions of Theorem B follow from either [DPU96, Hay99] or [SUZ11, combined with general arguments in [RRL12, §3].

Remark 1.1. The equilibrium states in Theorem $\mathrm{A}$ are found in [LRL13] using a result of Keller in [Kel85], showing that the corresponding transfer operator has a spectral gap in a certain Banach space containing the space of Lipschitz functions. On the other hand, the equilibrium states in Theorem $\mathrm{B}$ are found in SUZ11] through a Young tower with an exponential tail estimate. Thus, in both settings the equilibrium states satisfy several statistical properties, such as the Central Limit Theorem and the Almost Sure Invariance Principle, see [Kel85, Theorem 3.3] for the former and, e.g., Gou05, MN05, MN08, TK05] for the latter.

A natural question raised by Theorems $\mathrm{A}$ and $\mathrm{B}$ is what kind of phase transitions can occur for a smooth one dimensional map, on the space of Hölder continuous potentials. It is well-known that neutral periodic points 
are responsible for phase transitions on the space of Hölder continuous potentials of a sufficiently small exponent, see for example [Lop93, Theorem 3] or Sar01, Proposition 1]. A phase transition for a map without neutral periodic points would be more subtle to produce. "Fibonacci maps" are a natural candidate to have a phase transition. Given $\ell>1$ and a real parameter $c$, an interval map of the form $x \mapsto|x|^{\ell}+c$ is a Fibonacci map if, roughly speaking, the closest return times of the orbit of the critical point $x=0$ occur at the Fibonacci numbers, see for example [LM93, Theorem 1.1] for a precise definition in terms of kneading sequences. General results imply that for each real number $\ell>1$, there is a parameter $c(\ell)$ such that a suitable restriction $f_{\ell}$ of $x \mapsto|x|^{\ell}+c(\ell)$ is a Fibonacci map. For such $f_{\ell}$, the closure $\omega(\ell)$ of the critical orbit is a Cantor set, see for example [LM93, Theorem 1.2]. The results of [KN95] imply that there is $\ell_{1}>2$ such that for $\ell$ in $\left(0, \ell_{1}\right)$ the map $f_{\ell}$ satisfies the hypothesis of Theorem A. So for $\ell$ in $\left(0, \ell_{1}\right)$, the map $f_{\ell}$ does not have phase transitions in the space of Hölder continuous potentials.

Problem 1.2. Show that for every sufficiently large $\ell>1$, there are constants $C>0$ and $\alpha$ in $(0,1)$ such that the potential $\varphi_{0}(x):=-C \operatorname{dist}(x, \omega(\ell))^{\alpha}$ satisfies $P\left(f_{\ell}, \varphi_{0}\right)=0$.

A positive solution of this problem would imply that $\varphi_{0}$ is not hyperbolic for $f_{\ell}$, and that the function $t \mapsto P\left(f_{\ell}, t \varphi_{0}\right)$ is not real analytic.

1.2. Organization and strategy of the proof. To prove Theorems A and $\mathrm{B}$, we follow the general strategy of the proof of [RRL12, Main Theorem]. The main step in the proof of [IRRL12, Main Theorem] is the construction, for a given invariant measure with positive Lyapunov exponent, of an Iterated Function System (IFS) that is used to estimate the pressure from below. In this paper, we have to deal with the more difficult situation of an invariant measure of zero Lyapunov exponent. For such a measure, the construction of a suitable IFS does not seem possible. Instead, we consider a more general type of induced system, formed by multivalued functions. Once this induced system is constructed, we follow the strategy in IRRL12] to deduce our main results, although in the real case there are some extra difficulties coming from the fact that interval maps are not open maps in general.

The paper is organized as follows. In $\oint_{2}$ we state a version of Theorems $\mathrm{A}$ and $\mathrm{B}$ that holds for a more general class of maps; it is stated as "Main Theorem". After deriving Theorems A and B from the Main Theorem and known results in 2.1 , we state our main technical result as the "Key Lemma" in $\$ 2.2$, where we also derive the Main Theorem from it.

In 93 we construct an induced system formed by multivalued functions, and in $\$ 4$ we prove the Key Lemma using this induced system to estimate the pressure from below. 
1.3. Acknowledgments. The authors would like to thank Henk Bruin and Godofredo Iommi for useful comments on the first version.

\section{A REDUCTION}

We start this section stating a version of Theorems $\mathrm{A}$ and $\mathrm{B}$ that holds for a more general class of maps; it is stated as the "Main Theorem". In $\$ 2.1$ we derive Theorems $\mathrm{A}$ and $\mathrm{B}$ as a direct consequence of the Main Theorem and known results. In 92.2 we state our main technical result as the "Key Lemma", and we derive the Main Theorem from it.

To state the Main Theorem, we introduce a class of interval maps that includes non-degenerate smooth maps as special cases. Let $I$ be a compact interval. For a differentiable map $f: I \rightarrow I$, a point of $I$ is critical if the derivative of $f$ vanishes at it. Denote by $\operatorname{Crit}(f)$ the set of critical points of $f$. A differentiable interval map $f: I \rightarrow I$ is of class $C^{3}$ with non-flat critical points, if it has a finite number of critical points and if:

- The map $f$ is of class $C^{3}$ outside $\operatorname{Crit}(f)$;

- For each critical point $c$ of $f$ there exists a number $\ell_{c}>1$ and diffeomorphisms $\phi$ and $\psi$ of $\mathbb{R}$ of class $C^{3}$, such that $\phi(c)=\psi(f(c))=$ 0 , and such that on a neighborhood of $c$ on $I$, we have

$$
|\psi \circ f|=|\phi|^{\ell_{c}}
$$

Note that each smooth non-degenerate interval map is of class $C^{3}$ with nonflat critical points, and that each map of class $C^{3}$ with non-flat critical points is continuously differentiable.

For an interval map of class $C^{3}$ with non-flat critical points $f$, denote by $\operatorname{dom}(f)$ the interval on which $f$ is defined, and denote by dist the distance on $\operatorname{dom}(f)$ induced by the norm distance on $\mathbb{R}$. On the other hand, for a complex rational map $f$ we use $\operatorname{dom}(f)$ to denote the Riemann sphere $\overline{\mathbb{C}}$, which we endow with the spherical metric, that we also denote by dist. In both, the real and complex setting, for a subset $W$ of $\operatorname{dom}(f)$ we use $\operatorname{diam}(W)$ to denote the diameter of $W$ with respect to dist.

Definition 2.1. Let $f$ be either a complex rational map, or an interval map of class $C^{3}$ with non-flat critical points. The Julia set $J(f)$ of $f$ is the complement of the largest open subset of $\operatorname{dom}(f)$ on which the family of iterates of $f$ is normal.

If $f$ is a complex rational map of degree at least 2 , then $J(f)$ is a perfect set that is equal to the closure of repelling periodic points. Moreover, $J(f)$ is completely invariant and $f$ is topologically exact on $J(f)$. We denote by $\mathscr{A}_{\mathbb{C}}$ the collection of all rational maps of degree at least 2 .

In contrast with the complex setting, the Julia set of an interval map of class $C^{3}$ with non-flat critical points might be empty, reduced to a single 


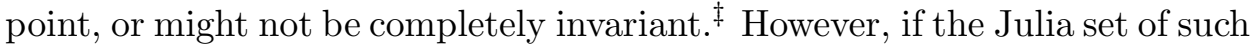
a map $f$ is not completely invariant, then it is possible to make an arbitrarily small smooth perturbation of $f$ outside a neighborhood of $J(f)$, so that the Julia set of the perturbed map is completely invariant and coincides with $J(f)$. We denote by $\mathscr{A} \mathbb{R}$ the collection of interval maps of class $C^{3}$ with non-flat critical points, whose Julia set contains at least 2 points and is completely invariant. Note that if $f: I \rightarrow I$ is a non-degenerate smooth map that is topologically exact, then $J(f)=I$ and $f$ is in $\mathscr{A} \mathbb{R}$. On the other hand, if $f$ is an interval map in $\mathscr{A}_{\mathbb{R}}$ that is topologically exact on $J(f)$, then $J(f)$ has no isolated points. For more background on the theory of Julia sets, see for example dMvS93] for the real setting, and [CG93, Mil06] for the complex setting.

Throughout the rest of this article we put $\mathscr{A}:=\mathscr{A} \mathbb{R} \cup \mathscr{A} \mathbb{C}$ and for each $f$ in $\mathscr{A}$ we restrict the action of $f$ to its Julia set. In particular, the topological pressure of $f$ is defined through measures supported on $J(f)$ and equilibrium states are supported on $J(f)$.

Definition 2.2. For $\beta>0$, a map $f$ in $\mathscr{A}$ satisfies the Polynomial Shrinking Condition with exponent $\beta$, if there exist constants $\rho_{0}>0$ and $C_{0}>0$ such that for every $x$ in $J(f)$, every integer $n \geq 1$, and every connected component $W$ of $f^{-n}(B(x, \rho))$, we have

$$
\operatorname{diam}(W) \leq C n^{-\beta} \text {. }
$$

Main Theorem. Let $\beta>1$, and let $f$ be a map in $\mathscr{A}$ satisfying the Polynomial Shrinking Condition with exponent $\beta$. Suppose furthermore in the real case that $f$ is topologically exact on its Julia set. Then for each $\alpha$ in $\left(\beta^{-1}, 1\right]$, every Hölder continuous potential $\varphi: J(f) \rightarrow \mathbb{R}$ of exponent $\alpha$ is hyperbolic for $f$.

Remark 2.3. Recall that a map $f$ in $\mathscr{A}$ satisfies the Topological ColletEckmann Condition, if there is a constant $\chi>0$ such that for every $\nu$ in $\mathcal{M}(J(f), f)$ we have $\int_{J(f)} \log |D f| d \nu \geq \chi$, see [PRLS03, RL12a] for other equivalent formulations. Each map in $\mathscr{A}$ that satisfies the Topological Collet-Eckmann condition satisfies the hypothesis of the Main Theorem for each $\beta>1$, see [RL12a, Main Theorem'] and [RL12b, Proposition 7.1] for the real case, and [PRLS03, Main Theorem] for the complex case.

Remark 2.4. Fix $\alpha$ in $(0,1)$, and consider the map $f_{\alpha}$ defined in 91.1 , Wellknown arguments show that $f_{\alpha}$ is topologically exact on $[0,1]$, and that $f_{\alpha}$ satisfies the Polynomial Shrinking Condition with exponent $\beta=1 / \alpha$. Being discontinuous, the map $f_{\alpha}$ is not in $\mathscr{A}$, so we cannot apply the Main Theorem directly to $f_{\alpha}$. However, using the Markov structure of $f_{\alpha}$ the arguments can be easily adapted to obtain that for each $\alpha^{\prime}$ in $(\alpha, 1]$, every Hölder continuous

\footnotetext{
${ }^{\ddagger}$ This last property can only happen if there is a turning point in the interior of the basin of a one-sided attracting neutral periodic point, that is eventually mapped to this neutral periodic point.
} 
potential of exponent $\alpha^{\prime}$ is hyperbolic for $f_{\alpha}$. The hypothesis that $\alpha^{\prime}$ is in $(\alpha, 1]$ is necessary: The potential $\varphi_{\alpha}:=-\log \left|D f_{\alpha}\right|$ is Hölder continuous of exponent $\alpha$, and it is not hyperbolic for $f_{\alpha}$. In fact, noting that $\varphi_{\alpha}(0)=0$ and $f_{\alpha}(0)=0$, for every integer $n \geq 1$ we have $\frac{1}{n} S_{n}\left(\varphi_{\alpha}\right)(0)=0$. Together with the equality $P\left(f_{\alpha}, \varphi_{\alpha}\right)=0$, shown for example in [Lop93, Theorem 3], this implies that $\varphi_{\alpha}$ is not hyperbolic for $f_{\alpha}$.

Given $f$ in $\mathscr{A}$, for each function $\varphi: J(f) \rightarrow \mathbb{R}$ and each integer $n \geq 1$, put

$$
S_{n}(\varphi):=\varphi+\varphi \circ f+\cdots+\varphi \circ f^{n-1} .
$$

Corollary 2.5. Let $\beta>1$, and let $f$ be a map in $\mathscr{A}$ satisfying the Polynomial Shrinking Condition with exponent $\beta$. Suppose furthermore in the real case that $f$ is topologically exact on its Julia set. Then for every $\alpha$ in $\left(\beta^{-1}, 1\right]$ and every Hölder continuous potential $\varphi$ of exponent $\alpha$, there is a unique equilibrium state $\nu$ of $f$ for the potential $\varphi$. Moreover, the measure-theoretic entropy of $\nu$ is positive and $\nu$ is exponentially mixing for $f$. Finally, for every Hölder function $\psi: J(f) \rightarrow \mathbb{R}$ of exponent $\alpha$, the function $t \mapsto P(f, \varphi+t \psi)$ is real analytic.

Proof. In the real case, the assertions are a direct consequence of the Main Theorem and of [LRL13, Theorem B]; for the real analyticity of $t \mapsto P(f, \varphi+$ $t \psi$ ), for each $t_{0}$ in $\mathbb{R}$ apply this result with $\varphi$ replaced by $\varphi+t_{0} \psi$.

To prove assertions in the complex setting, let $n \geq 1$ be an integer such that the function $\widetilde{\varphi}:=\frac{1}{n} S_{n}(\varphi)$ satisfies $\sup _{J(f)} \widetilde{\varphi}<P(f, \varphi)$. Since $f$ is Lipschitz as a self-map of $\overline{\mathbb{C}}$, the function $\widetilde{\varphi}$ is Hölder continuous of exponent $\alpha$. Moreover, $\widetilde{\varphi}$ is co-homologous to $\varphi$ : If we put

$$
h:=-\frac{1}{n} \sum_{j=0}^{n-1}(n-1-j) \varphi \circ f^{j},
$$

then $\widetilde{\varphi}=\varphi+h-h \circ f$. This implies that for every $\nu$ in $\mathcal{M}(J(f), f)$ we have $\int_{J(f)} \widetilde{\varphi} d \nu=\int_{J(f)} \varphi d \nu$, and therefore that $P(f, \widetilde{\varphi})=P(f, \varphi)$ and that $\widetilde{\varphi}$ and $\varphi$ share the same equilibrium states. An analogous argument shows that if we put $\widetilde{\psi}:=\frac{1}{n} S_{n}(\psi)$, then for every $t$ in $\mathbb{R}$ we have $P(\widetilde{\varphi}+t \widetilde{\psi})=$ $P(\varphi+t \psi)$. Thus

$$
\sup _{J(f)} \widetilde{\varphi}<P(f, \varphi)=P(f, \widetilde{\varphi})
$$

and for each equilibrium state $\nu$ of $f$ for the potential $\varphi$, we have

$h_{\nu}(f)=P(f, \varphi)-\int_{J(f)} \varphi d \nu=P(f, \widetilde{\varphi})-\int_{J(f)} \widetilde{\varphi} d \nu \geq P(f, \widetilde{\varphi})-\sup _{J(f)} \widetilde{\varphi}>0$.

On the other hand, the fact that $\nu$ is the unique equilibrium state of $f$ for the potential $\varphi$, and that $\nu$ is exponentially mixing, is obtained by applying Hay99 or SUZ11 to the potential $\widetilde{\varphi}$. To prove that the function $t \mapsto P(\varphi+t \psi)$ is real analytic, observe first that, when $\psi=\varphi$, SUZ11, Theorem 47 in $\S 6]$ asserts the function $t \mapsto P(\varphi+t \psi)$ is real analytic on a 
neighborhood of $t=0$. The proof of this result extends easily to the case $\psi$ is different from $\varphi$. To prove that $t \mapsto P(\varphi+t \psi)$ is real analytic on all of $\mathbb{R}$, for each $t_{0}$ in $\mathbb{R}$ apply this result with $\varphi$ replaced by $\varphi+t_{0} \psi$. This completes the proof that the proof of the corollary.

2.1. Proofs of Theorems $\mathrm{A}$ and $\mathrm{B}$ assuming the Main Theorem. We recall the "backward contracting property".

For each map $f$ in $\mathscr{A}$, put

$$
\mathrm{CV}(f):=f(\operatorname{Crit}(f)) \text { and } \operatorname{Crit}^{\prime}(f):=\operatorname{Crit}(f) \cap J(f) .
$$

For a subset $V$ of $\operatorname{dom}(f)$, and an integer $m \geq 1$, each connected component of $f^{-m}(V)$ is a pull-back of by $f^{m}$. For every $c$ in $\operatorname{Crit}(f)$ and $\delta>0$, denote by $\widetilde{B}(c, \delta)$ the pull-back of $B(f(c), \delta)$ by $f$ that contains $c$.

Definition 2.6. Given a constant $r>1$, a map $f$ in $\mathscr{A}$ is backward contracting with constant $r$, if there exists $\delta_{0}>0$ such that for every $c$ in $\operatorname{Crit}^{\prime}(f)$, every $\delta$ in $\left(0, \delta_{0}\right)$, every integer $n \geq 0$, and every component $W$ of $f^{-n}(\widetilde{B}(c, r \delta))$, we have that

$$
\operatorname{dist}(W, \mathrm{CV}(f)) \leq \delta \text { implies } \operatorname{diam}(W)<\delta .
$$

If for each $r>1$ the map $f$ is backward contracting with constant $r$, then $f$ is backward contracting.

A map $f$ in $\mathscr{A}$ is expanding away from critical points, if for every neighborhood $V$ of $\operatorname{Crit}^{\prime}(f)$ the map $f$ is uniformly expanding on the set

$$
K(V):=\left\{z \in J(f): f^{i}(z) \notin V \text { for all } i \geq 0\right\} .
$$

In other words, there exist $C>0$ and $\lambda>1$ such that for every $z$ in $K(V)$ and $n \geq 0$, we have $\left|D f^{n}(z)\right| \geq C \lambda^{n}$.

Fact 2.7 ([RLS10], Theorem A). For each map $f$ in $\mathscr{A}$ and each $\beta>0$, there exists $r>1$ such that the following property holds. If $f$ is backward contracting with constant $r$ and is expanding away from critical points, then $f$ satisfies the Polynomial Shrinking Condition with exponent $\beta$.

Proof of Theorem A. By [BRLSvS08, Theorem 1], the map $f$ is backward contracting and by Mañé's theorem $f$ is expanding away from critical points, see for example dMvS93. Then Fact 2.7 implies that for each $\beta>1$ the map $f$ satisfies the Polynomial Shrinking Condition with exponent $\beta$. So the desired assertions are a direct consequence of the Main Theorem and Corollary 2.5.

Proof of Theorem B. By either [LS10, Theorem A] or [RL07, Theorem A], the map $f$ is backward contracting, and by either [KvS09] or [RL07, Corollary 8.3], it is expanding away from critical points. Then Fact 2.7 implies that for each $\beta>1$ the map $f$ satisfies the Polynomial Shrinking Condition with exponent $\beta$. So the desired assertions are a direct consequence of the Main Theorem and Corollary 2.5. 
2.2. A reduction. In this section we prove the Main Theorem assuming the following lemma, whose proof occupies $\S \$ 3$, 4 ,

Key Lemma. Let $\beta>1$, and let $f$ be a map in $\mathscr{A}$ satisfying the Polynomial Shrinking of Components condition with exponent $\beta$. In the case $f$ is an interval map, assume furthermore that $f$ is topologically exact on its Julia set. Then for every $\alpha$ in $\left(\beta^{-1}, 1\right]$, every Hölder continuous function $\varphi$ : $J(f) \rightarrow \mathbb{R}$ of exponent $\alpha$, and every invariant ergodic probability measure $\nu$ supported on $J(f)$, there is a set of full measure of points $x_{0}$ such that

$$
\limsup _{n \rightarrow+\infty} \frac{1}{n} \log \sum_{y \in f^{-n}\left(x_{0}\right)} \exp \left(S_{n}(\varphi)(y)\right)>\int_{J(f)} \varphi d \nu .
$$

The proof of the Main Theorem is given after the following lemma. In the case $f$ is a complex rational map, the following lemma is [Prz90, Lemma 4]. The proof applies without change to the case where $f$ is an interval map.

Lemma 2.8. For each map $f$ in $\mathscr{A}$, and every continuous function $\varphi$ : $J(f) \rightarrow \mathbb{R}$, we have

$$
P(f, \varphi) \geq \limsup _{n \rightarrow+\infty} \frac{1}{n} \log \left(\sup _{x_{0} \in J(f)} \sum_{y \in f^{-n}\left(x_{0}\right)} \exp \left(S_{n} \varphi(y)\right)\right) .
$$

Proof of the Main Theorem assuming the Key Lemma. We show first

$$
\limsup _{n \rightarrow+\infty}\left(\sup _{J(f)} \frac{1}{n} S_{n}(\varphi)\right) \leq \sup _{\nu \in \mathcal{M}(J(f), f)} \int_{J(f)} \varphi d \nu .
$$

Since $J(f)$ is compact, for each integer $n \geq 1$ there is a point $x_{n}$ of $J(f)$ such that

$$
S_{n}(\varphi)\left(x_{n}\right)=\sup _{J(f)} S_{n}(\varphi)
$$

Put $\nu_{n}:=\frac{1}{n} \sum_{i=0}^{n-1} \delta_{f^{i}\left(x_{n}\right)}$, so that

$$
\int_{J(f)} \varphi d \nu_{n}=\frac{1}{n} S_{n}(\varphi)\left(x_{n}\right)=\sup _{J(f)} \frac{1}{n} S_{n}(\varphi) .
$$

It follows that there is a sequence positive integers $\left(n_{k}\right)_{k=1}^{+\infty}$ such that

$$
\lim _{k \rightarrow+\infty} \int_{J(f)} \varphi d \nu_{n_{k}}=\limsup _{n \rightarrow+\infty}\left(\sup _{J(f)} \frac{1}{n} S_{n}(\varphi)\right) .
$$

Let $\nu$ be an accumulation point of $\nu_{n_{k}}$ in the weak* topology. Then $\nu$ is in $\mathcal{M}(J(f), f)$, and

$$
\int_{J(f)} \varphi d \nu=\limsup _{n \rightarrow+\infty}\left(\sup _{J(f)} \frac{1}{n} S_{n}(\varphi)\right) .
$$

This proves (2.1). 
To prove that $\varphi$ is hyperbolic for $f$, let $\nu_{0}$ be an invariant probability measure maximizing the function $\nu \mapsto \int_{J(f)} \varphi d \nu$. Then for almost every ergodic component $\nu_{0}^{\prime}$ of $\nu_{0}$, we have $\int_{J(f)} \varphi d \nu_{0}^{\prime}=\int_{J(f)} \varphi d \nu_{0}$. Thus, the Key Lemma applied to such a $\nu_{0}^{\prime}$, together with Lemma 2.8 imply

$$
P(f, \varphi)>\int_{J(f)} \varphi d \nu_{0}^{\prime}=\int_{J(f)} \varphi d \nu_{0}=\sup _{\nu \in \mathcal{M}(J(f), f)} \int_{J(f)} \varphi d \nu .
$$

Together with (2.1), this implies that $\varphi$ is hyperbolic for $f$ and completes the proof of the Main Theorem.

\section{Iterated Multivalued Function Systems}

This section is devoted to the construction of an "Iterated Multivalued Function System", which is the main ingredient in the proof of the Key Lemma, compare with [IRRL12, $\S \S 5,6]$. It is stated as Proposition 3.1. below.

Given intervals $B$ and $W$ of $\mathbb{R}$, a multivalued function $\phi: B \rightarrow W$ is a function that to each point $x$ of $B$ associates a non-empty subset $\phi(x)$ of $W$. Note that each surjective function $f: W \rightarrow B$ defines a multivalued function $f^{-1}: B \rightarrow W$. If $\phi: B \rightarrow W$ and $\widetilde{\phi}: \widetilde{B} \rightarrow \widetilde{W}$ are multivalued functions such that $\widetilde{W}$ is contained in $B$, then the composition $\phi \circ \widetilde{\phi}$ of $\phi$ and $\widetilde{\phi}$ is the multivalued function $\phi \circ \widetilde{\phi}: \widetilde{B} \rightarrow W$ defined for $x$ in $\widetilde{B}$, by $(\phi \circ \widetilde{\phi})(x):=\bigcup_{y \in \widetilde{\phi}(x)} \phi(y)$. The composition of multivalued functions is easily seen to be associative.

Let $f$ be a map in $\mathscr{A}$. Given a compact and connected subset $B_{0}$ of $\operatorname{dom}(f)$ intersecting $J(f)$, a sequence multivalued functions $\left(\phi_{l}\right)_{l=1}^{+\infty}$ is an Iterated Multivalued Function System (IMFS) generated by $f$, if for every $l$ there is an integer $m_{l} \geq 1$, and a pull-back $W_{l}$ of $B_{0}$ by $f^{m_{l}}$ contained in $B_{0}$, such that

$$
f^{m_{l}}\left(W_{l}\right)=B_{0} \text { and } \phi_{l}=\left(\left.f^{m_{l}}\right|_{W_{l}}\right)^{-1} .
$$

In this case, $\left(m_{l}\right)_{l=1}^{+\infty}$ is the time sequence of $\left(\phi_{l}\right)_{l=1}^{+\infty}$, and $\left(\phi_{l}\right)_{l=1}^{+\infty}$ is defined on $B_{0}$. Note that for each subset $A$ of $B_{0}$ and each $l$, the set $\phi_{l}(A):=$ $f^{-m_{l}}(A) \cap W_{l}$ is non-empty.

Let $\left(\phi_{l}\right)_{l=1}^{+\infty}$ be an IMFS generated by $f$ with time sequence $\left(m_{l}\right)_{l=1}^{+\infty}$, defined on a set $B_{0}$. For each integer $n \geq 1$ put $\Sigma_{n}:=\{1,2, \ldots\}^{n}$ and denote the space of all finite words in the alphabet $\{1,2, \ldots$,$\} by \Sigma^{*}:=\bigcup_{n \geq 1} \Sigma_{n}$. For every integer $k \geq 1$ and $\underline{l}=l_{1} \cdots l_{k}$ in $\Sigma^{*}$, put

$$
|\underline{l}|=k, m_{\underline{l}}=m_{l_{1}}+m_{l_{2}}+\cdots+m_{l_{k}} \text {, and } \phi_{\underline{l}}=\phi_{l_{1}} \circ \cdots \circ \phi_{l_{k}} .
$$

Note that for every $x_{0}$ in $B_{0}$, and every pair of distinct words $\underline{l}$ and $\underline{l}^{\prime}$ in $\Sigma^{*}$ satisfying $m_{\underline{l}}=m_{\underline{l^{\prime}}}$, we have the following property:

$(*) \quad$ If the sets $\phi_{\underline{l}}\left(x_{0}\right)$ and $\phi_{\underline{l}^{\prime}}\left(x_{0}\right)$ intersect, then they coincide. 
The IMFS $\left(\phi_{l}\right)_{l=1}^{+\infty}$ is free, if there is $x_{0}$ in $B_{0}$ such that for every pair of distinct words $\underline{l}$ and $\underline{l}^{\prime}$ in $\Sigma^{*}$ such that $m_{\underline{l}}=m_{\underline{\underline{l}^{\prime}}}$, the sets $\phi_{\underline{l}}\left(x_{0}\right)$ and $\phi_{\underline{l}^{\prime}}\left(x_{0}\right)$ are disjoint.

Proposition 3.1. Let $\beta>1$, and let $f$ be a map in $\mathscr{A}$ satisfying the Polynomial Shrinking of Components condition with exponent $\beta$. In the case $f$ is an interval map, suppose furthermore that $f$ is topologically exact on its Julia set. Let $\alpha$ in $\left(\beta^{-1}, 1\right]$, let $\varphi: J(f) \rightarrow \mathbb{R}$ be Hölder continuous with exponent $\alpha$, and let $\nu$ be an ergodic invariant probability measure on $J(f)$ that is not supported on a periodic orbit. Then there exists a subset $X$ of $J(f)$ of full measure with respect to $\nu$, such that for every point $x_{0}$ in $X$ the following property holds: There exist $D>0$, a compact and connected subset $B_{0}$ of $\operatorname{dom}(f)$ containing $x_{0}$, and a free IMFS $\left(\phi_{l}\right)_{l=1}^{+\infty}$ generated by $f$ with time sequence $\left(m_{l}\right)_{l=1}^{+\infty}$, such that $\left(\phi_{l}\right)_{l=1}^{+\infty}$ is defined on $B_{0}$, and such that for every $l$ and every $y$ in $\phi_{l}\left(B_{0}\right)$ we have

$$
S_{m_{l}}(\varphi)(y) \geq m_{l} \int_{J(f)} \varphi d \nu-D .
$$

The proof of this proposition is at the end of this section.

Lemma 3.2. For each interval map $f: I \rightarrow I$ in $\mathscr{A}$ there is $\varepsilon>0$ such that the following property holds. Let $J_{0}$ be an interval contained in I satisfying $\left|J_{0}\right| \leq \varepsilon$, let $n \geq 1$ be an integer, and let $J$ be a pull-back of $J_{0}$ by $f^{n}$ whose closure is contained in the interior of I. Suppose in addition that for each $j$ in $\{1, \ldots, n\}$ the pull-back of $J_{0}$ by $f^{j}$ containing $f^{n-j}(J)$ has length bounded from above by $\varepsilon$. Then $f^{n}(\partial J) \subset \partial J_{0}$.

Proof. Note that each preimage of a point of $\partial I$ is either a point of $\partial I$, or a turning point in the interior of $I$. It follows that there is $\varepsilon$ in $(0,|I|)$ such that for every interval $J^{\prime}$ contained in $I$ that shares an endpoint with $I$ and satisfies $\left|J^{\prime}\right| \leq \varepsilon$, the following property holds: For each pull-back $J$ of $J^{\prime}$ by $f$, either $J$ shares an endpoint with $I$ and $f: J \rightarrow J^{\prime}$ is a bijection, or both endpoints of $J$ are mapped to the endpoint of $J^{\prime}$ that is not an endpoint of $I$. Reducing $\varepsilon$ if necessary, assume that for every pair of distinct elements $c$ and $c^{\prime}$ of $\operatorname{Crit}(f) \cup \partial I$, we have $\varepsilon<\operatorname{dist}\left(c, c^{\prime}\right)$. We prove the lemma for this choice of $\varepsilon$.

Let $J_{0}, n$, and $J$ be as in the statement of the lemma, and for each $j$ in $\{1, \ldots, n-1\}$ let $J_{j}$ be the pull-back of $J_{0}$ by $f^{j}$ containing $f^{n-j}(J)$. Note that $J_{n}=J$, and that our hypotheses imply that for each $j$ in $\{0, \ldots, n\}$ we have $\left|J_{j}\right| \leq \varepsilon$. We prove by induction that for each $j$ in $\{0, \ldots, n\}$, either $f^{j}\left(\partial J_{j}\right) \subset \partial J_{0}$, or $J_{j}$ shares an endpoint with $I$ and $f^{j}$ maps the endpoint of $J_{j}$ that is not an endpoint of $I$ to an endpoint of $J_{0}$; the lemma follows by taking $j=n$. The case $j=0$ being trivial, let $j$ in $\{1, \ldots, n\}$ be such that this property holds with $j$ replaced by $j-1$. If $J_{j-1}$ shares an endpoint with $I$, then the desired assertion follows from the induction hypothesis and our choice of $\varepsilon$. Suppose $J_{j-1}$ does not share an endpoint with $I$. If $J_{j}$ contains a turning point in its interior, then by our choice of $\varepsilon$ 
the interval $J_{j}$ does not contain any other turning point of $f$. It follows that both endpoints of $J_{j}$ are mapped to the same end point of $J_{j-1}$ by $f$. So by the induction hypothesis we have $f^{j}\left(\partial J_{j}\right) \subset \partial J_{0}$. It remains to consider the case where $J_{j}$ does not contain a turning point of $f$ in its interior. Then $f$ is injective on $J_{j}$. Thus, either $f: J_{j} \rightarrow J_{j-1}$ is a bijection, or $J_{j}$ shares an endpoint with $I$. In the former case we have $f^{j}\left(\partial J_{j}\right)=f^{j-1}\left(\partial J_{j-1}\right) \subset \partial J_{0}$. In the latter case, $f$ maps the endpoint $x_{j}$ of $J_{j}$ that is not an endpoint of $I$ to an endpoint of $J_{j-1}$, so by the induction hypothesis $f^{j}\left(x_{j}\right)$ is in $\partial J_{0}$. This completes the proof of the induction step and of the lemma.

Lemma 3.3. Let $f$ be an interval map in $\mathscr{A}$ that is topological exact on its Julia set, and let $x_{0}$ be a point of $J(f)$ such that $\left(x_{0},+\infty\right)\left(\operatorname{resp} .\left(-\infty, x_{0}\right)\right)$ intersects $J(f)$. Then for every open interval $U$ intersecting $J(f)$, and every sufficiently large integer $n \geq 1$, there is a point $y$ of $U$ in $f^{-n}\left(x_{0}\right)$ such that for every $\varepsilon>0$ the set $f^{n}(B(y, \varepsilon))$ intersects $\left(x_{0},+\infty\right)$ (resp. $\left.\left(-\infty, x_{0}\right)\right)$.

Proof. Using that $f$ is topological exact on its Julia set $J(f)$, we know that there is an integer $N \geq 1$ such that for every $n \geq N$ we have $f^{n}(U) \supset J(f)$. Fix $n \geq N$. Note that the set $f^{-n}\left(x_{0}\right)$ is finite, and there is a point $z$ of $U$ such that $f^{n}(z)$ is in $\left(x_{0},+\infty\right)$ (resp. $\left(-\infty, x_{0}\right)$ ). Let $y$ be a point of $f^{-n}\left(x_{0}\right)$ in $U$ such that for every $y^{\prime}$ of $f^{-n}\left(x_{0}\right)$ in $U$ we have $|y-z| \leq\left|y^{\prime}-z\right|$. Now let us prove the lemma holds for such $y$. In fact, otherwise, there is $\varepsilon_{0} \in(0,|y-z|)$ such that $f^{n}\left(B\left(y, \varepsilon_{0}\right)\right)$ is contained in $\left(-\infty, x_{0}\right]$ (resp. $\left.\left[x_{0},+\infty\right)\right)$. It follows that there is a point $z^{\prime}$ of $B\left(y, \varepsilon_{0}\right) \cap U$ such that $f^{n}\left(z^{\prime}\right)$ is in $\left(-\infty, x_{0}\right)$ (resp. $\left.\left(x_{0},+\infty\right)\right)$ and $\left|z^{\prime}-z\right|<|y-z|$. Since $f^{n}$ is continuous on $U$ it follows that there is $y^{\prime \prime}$ in $U$ such that $\left|y^{\prime \prime}-z\right|<|y-z|$ and $f^{n}\left(y^{\prime \prime}\right)=x_{0}$. This is a contradiction with our choice of $y$. The lemma is proved.

Lemma 3.4. Let $\beta>1$, and let $f$ be a map in $\mathscr{A}$ satisfying the Polynomial Shrinking Condition with exponent $\beta$. Then for every $\alpha$ in $\left(\beta^{-1}, 1\right]$ and every Hölder continuous function $\varphi: J(f) \rightarrow \mathbb{R}$ of exponent $\alpha$ there exist constants $\rho_{1}>0$ and $C_{1}>1$, such that for every point $z$ of $J(f)$, every integer $n \geq 1$, and every pull-back $W$ of $B\left(z, \rho_{1}\right)$ by $f^{n}$ the following holds: For every $x$ and $x^{\prime}$ in $W$ we have

$$
\left|S_{n}(\varphi)(x)-S_{n}(\varphi)\left(x^{\prime}\right)\right| \leq C_{1} .
$$

Proof. Let $C_{*} \geq 1$ be such that for every $z$ and $z^{\prime}$ in $J(f)$ we have

$$
\left|\varphi(z)-\varphi\left(z^{\prime}\right)\right| \leq C_{*} \operatorname{dist}\left(z, z^{\prime}\right)^{\alpha} .
$$

Noticing that $f$ satisfies the Polynomial Shrinking Condition with exponent $\beta$, there exist constants $\rho_{0}>0$ and $C_{0}>1$ such that for every point $z$ in $J(f)$, every integer $n \geq 1$, and every pull-back $W^{\prime}$ of $B\left(z, \rho_{0}\right)$ by $f^{n}$, we have $\operatorname{diam}\left(W^{\prime}\right) \leq C_{0} n^{-\beta}$. Therefore, for every integer $n \geq 1$, every point $z$ in $J(f)$ and every pair of points $x$ and $x^{\prime}$ in the same pull-back $W$ of $B\left(z, \rho_{0}\right)$ 
by $f^{n}$, we have

$$
\begin{aligned}
\left|S_{n}(\varphi)(x)-S_{n}(\varphi)\left(x^{\prime}\right)\right| & =\left|\sum_{i=0}^{n-1} \varphi\left(f^{i}(x)\right)-\sum_{i=0}^{n-1} \varphi\left(f^{i}\left(x^{\prime}\right)\right)\right| \\
& \leq \sum_{i=0}^{n-1} C_{*} \operatorname{diam}\left(f^{i}(W)\right)^{\alpha} \\
& \leq \sum_{i=0}^{n-1} C_{*} C_{0}^{\alpha}(n-i)^{-\beta \alpha} \\
& \leq C_{*} C_{0}^{\alpha} \sum_{m=1}^{+\infty} m^{-\beta \alpha} .
\end{aligned}
$$

This proves the lemma with constants $\rho_{1}=\rho_{0}$ and $C_{1}=C_{*} C_{0}^{\alpha} \sum_{m=1}^{+\infty} m^{-\beta \alpha}$.

Lemma 3.5. Let $f$ be a map in $\mathscr{A}$, let $\varphi: J(f) \rightarrow \mathbb{R}$ be a continuous function, and let $\nu$ be an invariant and ergodic Borel probability measure supported on $J(f)$. Then there exists a Borel subset $X^{\prime}$ of $J(f)$ of full measure with respect to $\nu$, such that the following holds. For every $D^{\prime}>0$, and every point $x$ of $X^{\prime}$ there exist a strictly increasing sequence positive integers $\left(n_{l}\right)_{l=1}^{+\infty}$ such that for every $l$ we can choose a point $x_{l}$ in $f^{-n_{l}}(x)$ so that:

1. $x_{l+1}$ is in $f^{-\left(n_{l+1}-n_{l}\right)}\left(x_{l}\right)$;

2. $S_{n_{l}}(\varphi)\left(x_{l}\right) \geq n_{l} \int_{J(f)} \varphi d \nu-D^{\prime}$.

To prove this lemma we use the natural extension of $\left.f\right|_{J(f)}$, that we proceed to recall. Let $\mathbb{Z}_{-}$denote the set of all non-positive integers and endow

$$
\mathcal{Z}:=\left\{\left(z_{m}\right)_{m \in \mathbb{Z}_{-}} \in J(f)^{\mathbb{Z}_{-}}: \text {for every } m \in \mathbb{Z}_{-}, f\left(z_{m-1}\right)=z_{m}\right\}
$$

with the product topology. Define $T: \mathcal{Z} \rightarrow \mathcal{Z}$ by

$$
T\left(\left(\cdots, z_{-2}, z_{-1}, z_{0}\right)\right)=\left(\cdots, z_{-2}, z_{-1}, z_{0}, f\left(z_{0}\right)\right)
$$

and $\pi: \mathcal{Z} \rightarrow J(f)$ by $\pi\left(\left(z_{m}\right)_{m \in \mathbb{Z}_{-}}\right)=z_{0}$. Note that $T$ is a bijection, $T^{-1}$ is measurable, $\pi$ is continuous and onto, and $\pi \circ T=f \circ \pi$. We call $(\mathcal{Z}, T)$ the natural extension of $(J(f), f)$. If $\nu$ is a Borel probability measure on $J(f)$ that is invariant and ergodic for $f$, then there exists a unique Borel probability measure $\widetilde{\nu}$ on $\mathcal{Z}$ that is invariant and ergodic for $T$, and that satisfies $\pi_{*} \widetilde{\nu}=\nu$, see for example [PU10, §2.7].

The following is a well-known consequence of the pointwise ergodic theorem, see for example [PRLS04, Lemma 1.3] for a proof.

Lemma 3.6. Let $(\mathcal{Z}, \mathscr{B}, \widetilde{\nu})$ be a probability space, and let $T: \mathcal{Z} \rightarrow \mathcal{Z}$ be an ergodic measure preserving transformation. Then for each function 
$\psi: \mathcal{Z} \rightarrow \mathbb{R}$ that is integrable with respect to $\widetilde{\nu}$, there exists a subset $Z$ of $\mathcal{Z}$ such that $\widetilde{\nu}(Z)=1$, and such that for every $\underline{z}$ in $Z$ we have

$$
\limsup _{n \rightarrow+\infty} \sum_{i=0}^{n-1}\left(\psi\left(T^{i}(\underline{z})\right)-\int_{\mathcal{Z}} \psi d \widetilde{\nu}\right) \geq 0 .
$$

Proof of Lemma 3.5. Let $(\mathcal{Z}, T)$ and $\widetilde{\nu}$ be as above, and note that $\widetilde{\nu}$ is also ergodic with respect to $T^{-1}$. Applying Lemma 3.6 to the continuous function $\psi=\varphi \circ \pi$, we obtain that there exists a subset $Z$ of $\mathcal{Z}$ of full measure with respect to $\widetilde{\nu}$, such that for every point $\left(z_{m}\right)_{m \in \mathbb{Z}_{-}}$in $Z$ we have

$$
\limsup _{n \rightarrow+\infty} \sum_{i=0}^{n-1}\left(\varphi \circ \pi\left(T^{-i}\left(\left(z_{m}\right)_{m \in \mathbb{Z}_{-}}\right)\right)-\int_{\mathcal{Z}} \varphi \circ \pi d \widetilde{\nu}\right) \geq 0 .
$$

Note that the set $X^{\prime}:=\pi(Z)$ satisfies

$$
\nu\left(X^{\prime}\right)=\widetilde{\nu}\left(\pi^{-1}(\pi(Z))\right) \geq \widetilde{\nu}(Z)=1,
$$

so $\nu\left(X^{\prime}\right)=1$. To verify that $X^{\prime}$ satisfies the desired properties, let $D^{\prime}>0$, let $x$ be a point of $X^{\prime}$, and choose a point $\left(z_{m}\right)_{m \in \mathbb{Z}_{-}}$of $Z$ such that $\pi\left(\left(z_{m}\right)_{m \in \mathbb{Z}_{-}}\right)=$ $x$. Then there is a strictly increasing sequence positive integers $\left(n_{l}\right)_{l=1}^{+\infty}$ such that for every $l$ we have

$$
\sum_{i=0}^{n_{l}-1} \varphi \circ \pi\left(T^{-i}\left(\left(z_{m}\right)_{m \in \mathbb{Z}_{-}}\right)\right) \geq n_{l} \int_{J(f)} \varphi d \nu-D^{\prime} .
$$

For each integer $l \geq 1$ put

$$
x_{l}:=\pi\left(T^{-n_{l}}\left(\left(z_{m}\right)_{m \in \mathbb{Z}_{-}}\right)\right)=z_{n_{l}} \in f^{-n_{l}}(z) .
$$

The first part is then a direct consequence of the definitions, and the second follows from (3.2).

Proof of Proposition 3.1. In the real case, denote by $I$ the domain of $f$.

Let $X^{\prime}$ be the subset of $J(f)$ given by Lemma 3.5, and let $X$ be the complement in $X^{\prime}$ of the set of preperiodic points of $f$. Since $\nu$ is ergodic and it is not supported on a periodic orbit, the set $X$ has full measure for $\nu$. Fix a point $x_{0}$ of $X$. In the real case assume $x_{0}$ is not an endpoint of $I$.

Let $\varepsilon>0$ be the constant given by Lemma 3.2, and let $\rho_{1}>0$ and $C_{1}>1$ be the constants given by Lemma 3.4. Moreover, let $\rho_{0}>0$ and $C_{0}>1$ be such that for every $z$ in $J(f)$, every integer $n \geq 1$, and every pull-back $W$ of $B\left(z, \rho_{0}\right)$ by $f^{n}$, we have

$$
\operatorname{diam}(W) \leq \min \left\{C_{0} n^{-\beta}, \varepsilon\right\}
$$

Fix $\rho$ in $\left(0, \min \left\{\rho_{0}, \rho_{1}\right\}\right)$. In the real case, assume in addition that $\rho<$ $\operatorname{dist}\left(x_{0}, \partial I\right)$.

In part 1 below we define the IMFS, and in part 2 we show it is free and that it satisfies (3.1).

1. Let $D^{\prime},\left(n_{l}\right)_{l=1}^{+\infty}$, and $\left(x_{l}\right)_{l=1}^{+\infty}$ be given by Lemma 3.5 with $x=x_{0}$. Taking a subsequence if necessary, assume $\left(x_{l}\right)_{l=1}^{+\infty}$ converges to a point $w_{0}$. In 
the complex case, using that $f$ is topologically exact on $J(f)$, there exist an integer $M \geq 1$ and distinct points $y_{0}$ and $y_{1}$ of $f^{-M}\left(w_{0}\right)$ in $B\left(x_{0}, \rho\right)$. Let $\rho^{\prime}$ in $(0,+\infty)$ be such that the pull-backs $U_{0}$ and $U_{1}$ of $B\left(w_{0}, \rho^{\prime}\right)$ by $f^{M}$ containing $y_{0}$ and $y_{1}$, respectively, are disjoint and contained in $B\left(x_{0}, \rho\right)$. Note that, since in the complex case the map $f$ is open, for every sufficiently large $l$ the point $x_{l}$ is contained in $f^{M}\left(U_{0}\right)$ and in $f^{M}\left(U_{1}\right)$. In the real case, assume without loss of generality that for infinitely many integers $l$ the point $x_{l}$ satisfies $x_{l}>w_{0}$. Note that $x_{0}$ is not in the boundary of a periodic Fatou component, since by hypothesis $x_{0}$ is not preperiodic. Therefore we can assume that there are 2 disjoint open intervals $\widetilde{U}_{0}$ and $\widetilde{U}_{1}$ in $\left(x_{0}-\rho, x_{0}\right)$, each of them intersecting $J(f)$. Since $f$ is topologically exact on $J(f)$ and $w_{0}$ is in $J(f)$, by Lemma 3.3 there is an integer $M \geq 1$, and distinct points $y_{0}$ and $y_{1}$ of $f^{-M}\left(w_{0}\right)$ in $\widetilde{U}_{0}$ and $\widetilde{U}_{1}$, respectively, such that for every $\varepsilon>0$ each of the sets $f^{M}\left(B\left(y_{0}, \varepsilon\right)\right)$ and $f^{M}\left(B\left(y_{1}, \varepsilon\right)\right)$ intersects $\left(w_{0},+\infty\right)$. Let $\rho^{\prime}>0$ be such that the pull-backs $U_{0}$ and $U_{1}$ of $B\left(w_{0}, \rho^{\prime}\right)$ by $f^{M}$ containing $y_{0}$ and $y_{1}$, respectively, are contained in $\widetilde{U}_{0}$ and $\widetilde{U}_{1}$, respectively. It follows that $U_{0}$ and $U_{1}$ are disjoint and contained in $\left(x_{0}-\rho, x_{0}\right)$ and that for infinitely many $l$ the point $x_{l}$ is contained in $f^{M}\left(U_{0}\right)$ and in $f^{M}\left(U_{1}\right)$. Using the Polynomial Shrinking Condition and taking a subsequence if necessary, assume in both, the real and complex cases, that for every integer $l \geq 1$ we have $n_{l+1}-n_{l} \geq$ $M$, that the point $x_{l}$ is contained in $f^{M}\left(U_{0}\right)$ and in $f^{M}\left(U_{1}\right)$, and that the pull-back $W_{l}$ of $\overline{B\left(x_{0}, \rho\right)}$ by $f^{n_{l}}$ containing $x_{l}$ is contained in $B\left(w_{0}, \rho^{\prime}\right)$. Interchanging $y_{0}$ and $y_{1}$, and taking a subsequence if necessary, assume that for every $l$ the point $f^{n_{l+1}-n_{l}-M}\left(x_{l+1}\right)$ is not in $U_{0}$. For each $l$ choose a pull-back $W_{l}^{\prime}$ of $W_{l}$ by $f^{M}$ that contains a point $x_{l}^{\prime}$ of $f^{-M}\left(x_{l}\right)$ and that is contained in $U_{0}$.

In the complex case put

$$
B_{0}:=\overline{B\left(x_{0}, \rho\right)}, M^{\prime}:=M, U_{0}^{\prime}:=U_{0},
$$

and for each $l$ put $W_{l}^{\prime \prime}:=W_{l}^{\prime}$. Note that for each $l$ we have

$$
W_{l}^{\prime \prime} \subset U_{0}^{\prime} \subset B_{0} \text { and } f^{n_{l}+M^{\prime}}\left(W_{l}^{\prime \prime}\right)=B_{0} .
$$

In the real case, note that $W_{l}^{\prime}$ is contained in $U_{0} \subset B\left(x_{0}, \rho\right)$, so the closure of $W_{l}^{\prime}$ is contained in the interior of $I$. So by Lemma 3.2 the set $f^{n_{l}+M}\left(\partial W_{l}^{\prime}\right)$ is contained in $\partial B\left(x_{0}, \rho\right)$. Thus, for each $l$ the set $f^{n_{l}+M}\left(W_{l}^{\prime}\right)$ contains either $\left[x_{0}-\rho, x_{0}\right]$ or $\left[x_{0}, x_{0}+\rho\right]$. Suppose there are infinitely many $l$ such that $f^{n_{l}+M}\left(W_{l}^{\prime}\right)$ contains $\left[x_{0}-\rho, x_{0}\right]$. Taking a subsequence if necessary, assume this holds for every $l$. Then for every $l$ there is a pull-back $W_{l}^{\prime \prime}$ of $\left[x_{0}-\rho, x_{0}\right]$ by $f^{n_{l}+M}$ that is contained in $W_{l}^{\prime}$ and such that $f^{n_{l}+M}\left(W_{l}^{\prime \prime}\right)=$ $\left[x_{0}-\rho, x_{0}\right]$. In this case we put

$$
B_{0}:=\left[x_{0}-\rho, x_{0}\right], M^{\prime}:=M, \text { and } U_{0}^{\prime}:=U_{0},
$$

and note that $W_{l}^{\prime \prime} \subset W_{l}^{\prime} \subset U_{0}^{\prime} \subset\left[x_{0}-\rho, x_{0}\right]=B_{0}$. It remains to consider the case where for each $l$, outside finitely many exceptions, the set $f^{n_{l}+M}\left(W_{l}^{\prime}\right)$ contains $\left[x_{0}, x_{0}+\rho\right]$, but it does not contain $\left[x_{0}-\rho, x_{0}\right]$. Taking a subsequence 
if necessary, assume this holds for every $l$. Since $x_{0}$ is not in the boundary of a Fatou component, by Lemma 3.3 there is an integer $\widetilde{M} \geq 1$ and a pullback $U_{0}^{\prime}$ of $U_{0}$ by $f^{\widetilde{M}}$ that is contained in $\left(x_{0}, x_{0}+\rho\right)$, and such that for infinitely many $l$ the point $x_{l}^{\prime}$ is contained in $f^{\widetilde{M}}\left(U_{0}^{\prime}\right)$. Taking a subsequence if necessary, assume that for every $l$ we have $n_{l+1}-n_{l} \geq M+\widetilde{M}$, and that the point $x_{l}^{\prime}$ is contained in $f^{\widetilde{M}}\left(U_{0}^{\prime}\right)$. Since for each $l$ the point $f^{n_{l+1}-n_{l}-M}\left(x_{l+1}\right)$ is not in $U_{0}$, it follows that the point $f^{n_{l+1}-n_{l}-M-\widetilde{M}}\left(x_{l+1}\right)$ is not in $U_{0}^{\prime}$. For each $l$ choose a pull-back $\widetilde{W_{l}^{\prime}}$ of $W_{l}^{\prime}$ by $f^{\widetilde{M}}$ contained in $U_{0}^{\prime}$ and that contains a point of $f^{-\widetilde{M}}\left(x_{l}^{\prime}\right)$. By Lemma 3.2, the set $f^{n_{l}+M+\widetilde{M}}\left(\partial \widetilde{W}_{l}^{\prime}\right)$ is contained in $\partial B\left(x_{0}, \rho\right)$. Since the set $f^{n_{l}+M+\widetilde{M}}\left(\widetilde{W}_{l}^{\prime}\right)$ is contained in $f^{n_{l}+M}\left(W_{l}^{\prime}\right)$ and this last set does not contain $\left[x_{0}-\rho, x_{0}\right]$, we conclude that $f^{n_{l}+M+\widetilde{M}}$ maps both endpoints of $\widetilde{W}_{l}^{\prime}$ to $x=x_{0}+\rho$. Since by construction $f^{n_{l}+M+\widetilde{M}}\left(\widetilde{W}_{l}^{\prime}\right)$ contains $x=x_{0}$, we conclude that $f^{n_{l}+M+\widetilde{M}}\left(\widetilde{W}_{l}^{\prime}\right)$ contains $\left[x_{0}, x_{0}+\rho\right]$. So there is a pull-back $W_{l}^{\prime \prime}$ of $\left[x_{0}, x_{0}+\rho\right]$ by $f^{n_{l}+M+\widetilde{M}}$ that is contained in $\widetilde{W}_{l}^{\prime}$, and such that $f^{n_{l}+M+\widetilde{M}}\left(W_{l}^{\prime \prime}\right)=\left[x_{0}, x_{0}+\rho\right]$. Note that $W_{l}^{\prime \prime} \subset \widetilde{W}_{l}^{\prime} \subset U_{0}^{\prime} \subset$ $\left(x_{0}, x_{0}+\rho\right)$. In this case we put $B_{0}:=\left[x_{0}, x_{0}+\rho\right]$, and $M^{\prime}:=M+\widetilde{M}$.

In both, the real and complex cases, for each $l$ we put

$$
\phi_{l}:=\left(\left.f^{n_{l}+M^{\prime}}\right|_{W_{l}^{\prime \prime}}\right)^{-1} .
$$

Then, $\left(\phi_{l}\right)_{l=1}^{+\infty}$ is an IMFS generated by $f$ with time sequence $\left(m_{l}\right)_{l=0}^{+\infty}:=$ $\left(n_{l}+M^{\prime}\right)_{l=0}^{+\infty}$ that is defined on $B_{0}$. Moreover, for each $l$ we have

$$
n_{l+1}-n_{l} \geq M^{\prime}, W_{l}^{\prime \prime} \subset U_{0}^{\prime} \text {, and } f^{n_{l+1}-n_{l}-M^{\prime}}\left(x_{l+1}\right) \notin U_{0}^{\prime} .
$$

2. To prove that the IMFS $\left(\phi_{l}\right)_{l=1}^{+\infty}$ is free, let $k \geq 1$ and $k^{\prime} \geq 1$ be integers and let

$$
\underline{l}:=l_{1} l_{2} \cdots l_{k} \text { and } \underline{l}^{\prime}:=l_{1}^{\prime} l_{2}^{\prime} \cdots l_{k^{\prime}}^{\prime}
$$

be different words in $\Sigma^{*}$ such that $m_{\underline{l}}=m_{\underline{l}^{\prime}}$. Assume without loss of generality that $l_{k^{\prime}}^{\prime} \geq l_{k}+1$. Note that the set

$$
f^{m_{\underline{l}}-m_{l_{k}}}\left(\phi_{\underline{l}}\left(x_{0}\right)\right)=\phi_{l_{k}}\left(x_{0}\right) .
$$

is contained $W_{l_{k}}^{\prime \prime}$, and therefore in $U_{0}^{\prime}$. On the other hand, we have

$$
m_{l_{k^{\prime}}^{\prime}}-m_{l_{k}}=n_{l_{k^{\prime}}^{\prime}}-n_{l_{k}} \geq n_{l_{k}+1}-n_{l_{k}} \geq M^{\prime}
$$

and therefore the set

$$
\begin{aligned}
& f^{m_{\underline{l}}-m_{l_{k}}}\left(\phi_{\underline{l}^{\prime}}\left(x_{0}\right)\right)=f^{m_{\underline{l}^{\prime}}-m_{l_{k}}}\left(\phi_{{\underline{l^{\prime}}}^{\prime}}\left(x_{0}\right)\right)=f^{m_{l_{k^{\prime}}^{\prime}}-m_{l_{k}}}\left(\phi_{l_{k^{\prime}}}\left(x_{0}\right)\right) \\
& =f^{m_{l_{k^{\prime}}^{\prime}}-m_{l_{k}}-M^{\prime}}\left(\left(f^{\left.n_{l_{k^{\prime}}}\right|_{W_{l_{k^{\prime}}}}}\right)^{-1}\left(x_{0}\right)\right)
\end{aligned}
$$


contains the point

$$
f^{m_{l^{\prime}{ }^{\prime}}-m_{l_{k}}-M^{\prime}}\left(x_{l_{k^{\prime}}^{\prime}}\right)=f^{n_{l^{\prime}},-n_{l_{k}}-M^{\prime}}\left(x_{l_{k^{\prime}}^{\prime}}\right)=f^{n_{l_{k}+1}-n_{l_{k}}-M^{\prime}}\left(x_{l_{k}+1}\right) .
$$

By construction this point is not in $U_{0}^{\prime}$, so we conclude that the sets

$$
f^{m_{\underline{l}}-m_{l_{k}}}\left(\phi_{\underline{\underline{l}}}\left(x_{0}\right)\right) \text { and } f^{m_{\underline{\underline{l}}}-m_{l_{k}}}\left(\phi_{{\underline{l^{\prime}}}^{\prime}}\left(x_{0}\right)\right)
$$

are different. This implies that the sets $\phi_{\underline{l}}\left(x_{0}\right)$ and $\phi_{\underline{l}^{\prime}}\left(x_{0}\right)$ are different, and by property $(*)$ stated above the statement of the proposition, that they are disjoint. This completes the proof that the IMFS $\left(\phi_{l}\right)_{l=1}^{+\infty}$ is free.

Finally, let us check inequality (3.1) in the statement of the proposition. Recall that for every $l$ and $y$ in $\phi_{l}\left(B_{0}\right)$, the point $f^{M^{\prime}}(y)$ is in $W_{l}$. Thus, by Lemma 3.4 and by part 2 of Lemma 3.5 we have

$$
\begin{aligned}
S_{m_{l}}(\varphi)(y) & =S_{n_{l}}(\varphi)\left(f^{M^{\prime}}(y)\right)+S_{M^{\prime}}(\varphi)(y) \\
& \geq S_{n_{l}}(\varphi)\left(x_{l}\right)-C_{1}+S_{M^{\prime}}(\varphi)(y) \\
& \geq n_{l} \int_{J(f)} \varphi d \nu-D^{\prime}-C_{1}+S_{M^{\prime}}(\varphi)(y) \\
& \geq m_{l} \int_{J(f)} \varphi d \nu-D^{\prime}-C_{1}-2 M^{\prime} \sup _{J(f)} \varphi .
\end{aligned}
$$

This proves (3.1) with $D=D^{\prime}+C_{1}+2 M^{\prime} \sup _{J(f)} \varphi$, and completes the proof of the proposition.

\section{Proof of the Key Lemma}

In this section we complete the proof of the Key Lemma. The case where the measure $\nu$ is supported on a periodic orbit is different. For the complex case we refer to IRRL12, Proposition 4.1]. The proof of [IRRL12, Proposition 4.1] does not apply directly to interval maps, as it uses that complex rational maps are open as maps acting on $\overline{\mathbb{C}}$. The case of interval maps is treated in Lemma 4.1, below. The proof of the Key Lemma is completed after this lemma.

Lemma 4.1. Let $\beta>1$, and let $f$ be an interval map in $\mathscr{A}$ that is topologically exact on its Julia set, and that satisfies the Polynomial Shrinking of Components condition with exponent $\beta$. Then for every $\alpha$ in $\left(\beta^{-1}, 1\right]$, every Hölder continuous function $\varphi: J(f) \rightarrow \mathbb{R}$ with exponent $\alpha$, and every periodic point $x_{0}$ in $J(f)$ of $f$ of period $N$, we have

$$
\limsup _{n \rightarrow+\infty} \frac{1}{n} \log \sum_{y \in f^{-n}\left(x_{0}\right)} \exp \left(S_{n}(\varphi)(y)\right)>\frac{1}{N} S_{N}(\varphi)\left(x_{0}\right) .
$$

Proof. Let $\rho_{0}>0$ and $C_{0}>1$ be such that for every $z$ in $J(f)$, every integer $n \geq 1$, and every pull-back $W$ of $B\left(z, \rho_{0}\right)$ by $f^{n}$, we have $\operatorname{diam}(W) \leq C_{0} n^{-\beta}$. The proof is divided in 2 parts. In part 1 we construct an induced map, and in part 2 we show an inequality analogous to (4.1) for the induced map, from which (4.1) follows as a direct consequence. 
1. Fix a periodic point $x_{0}$ in $J(f)$ of period $N$. Since $\left|\left(f^{N}\right)^{\prime}\left(x_{0}\right)\right| \geq 1$, we know that there is $\rho$ in $\left(0, \rho_{0}\right)$ such that there is a local inverse $\phi$ of $f^{2 N}$ defined on $B\left(x_{0}, \rho\right)$ and fixing $x_{0}$. Note that $f^{2 N} \circ \phi$ is the identity map on $B\left(x_{0}, \rho\right)$, hence $\phi$ is increasing on $B\left(x_{0}, \rho\right)$, and $f^{2 N}$ is increasing on $\phi\left(B\left(x_{0}, \rho\right)\right)$. Since $x_{0}$ is in $J(f)$, changing orientation and reducing $\rho$ if necessary, assume that $\left(x_{0}, x_{0}+\rho / 2\right)$ intersects $J(f)$, and that for every $y$ in $\left(x_{0}, x_{0}+\rho\right)$ we have $\phi(y)<y$. Since $f$ is topologically exact on its Julia set, by Lemma 3.3 there exist an integer $k^{\prime} \geq 1$, and a point $z^{\prime}$ in $\left(x_{0}, x_{0}+\rho / 2\right)$, such that $f^{2 N k^{\prime}}\left(z^{\prime}\right)=x_{0}$, and such that for every $\varepsilon>0$ the set $f^{2 N k^{\prime}}\left(B\left(z^{\prime}, \varepsilon\right)\right)$ intersects $\left(x_{0}, x_{0}+\rho / 2\right)$. Fix $\varepsilon$ in $\left(0,\left|z^{\prime}-x_{0}\right|\right)$ such that $f^{2 N k^{\prime}}\left(B\left(z^{\prime}, \varepsilon\right)\right) \subset B\left(x_{0}, \rho / 2\right)$. Note that the closure of $B\left(z^{\prime}, \varepsilon\right)$ is contained in $\left(x_{0}, x_{0}+\rho\right)$.

Let $W$ be the pull-back of $f^{2 N k^{\prime}}\left(B\left(z^{\prime}, \varepsilon\right)\right) \cap\left[x_{0}, x_{0}+\rho / 2\right)$ by $f^{2 N k^{\prime}}$ containing $z^{\prime}$. Since $f^{2 N k^{\prime}}$, and hence $\phi^{k^{\prime}}$, is continuous, reducing $\varepsilon$ if necessary, assume that $U_{0}^{\prime}:=\phi^{k^{\prime}}\left(f^{2 N k^{\prime}}(W)\right)$ is disjoint from $\bar{W}$. By our choice of $\phi$, and the hypothesis that $f$ satisfies the Polynomial Shrinking of Components condition, we know that for every $x$ in $\left(x_{0}, x_{0}+\rho\right)$ we have $\lim _{k \rightarrow+\infty} \operatorname{diam}\left(\phi^{k}\left(\left[x_{0}, x\right]\right)\right)=0$. This implies that for every interval $U \subset\left[x_{0}, x_{0}+\rho\right)$ we have

$$
\lim _{k \rightarrow+\infty} \operatorname{diam}\left(\phi^{k}(U)\right)=0 \text { and } \lim _{k \rightarrow+\infty} \operatorname{dist}\left(\phi^{k}(U), x_{0}\right)=0 .
$$

Noting that

$$
W \subset B\left(z^{\prime}, \varepsilon\right) \subset\left(x_{0}, x_{0}+\rho\right) \text { and } x_{0} \in f^{2 N k^{\prime}}(W) \subset\left[x_{0}, x_{0}+\rho / 2\right),
$$

it follows that there is $k_{1} \geq 0$ such that

$$
U_{1}:=\phi^{k_{1}}(W) \subset f^{2 N k^{\prime}}(W),
$$

and

$$
\operatorname{diam}\left(\phi^{k_{1}+k^{\prime}}\left(f^{2 N k^{\prime}}(W)\right)\right)<\operatorname{diam}\left(f^{2 N k^{\prime}}(W)\right) .
$$

Put $k_{0}:=k_{1}+k^{\prime}$ and $U_{0}:=\phi^{k_{1}}\left(U_{0}^{\prime}\right)$. Then we have

$$
k_{0} \geq 1, U_{0} \cap U_{1}=\emptyset \text {, and } U_{1} \subset f^{2 N k^{\prime}}(W) .
$$

By (4.3) and the fact that $f^{2 N k^{\prime}}(W)$ contains $x_{0}$, the set

$$
U_{0}=\phi^{k_{1}}\left(U_{0}^{\prime}\right)=\phi^{k_{0}}\left(f^{2 N k^{\prime}}(W)\right)
$$

is contained in $f^{2 N k^{\prime}}(W)$. Finally, note that

$$
f^{2 N k_{0}}\left(U_{1}\right)=f^{2 N k^{\prime}}(W)=f^{2 N k_{0}}\left(U_{0}\right) .
$$

Put

$$
U:=U_{0} \cup U_{1} \text { and } \widehat{f}:=\left.f^{2 N k_{0}}\right|_{U} .
$$

2. Put $\widehat{\varphi}:=\frac{1}{2 N k_{0}} S_{2 N k_{0}}(\varphi)$, for every integer $m \geq 1$ put

$$
\widehat{S}_{m}(\widehat{\varphi}):=\widehat{\varphi}+\widehat{\varphi} \circ \widehat{f}+\cdots+\widehat{\varphi} \circ \widehat{f}^{m-1},
$$


and note that to prove the lemma it suffices to show

$$
\limsup _{m \rightarrow+\infty} \frac{1}{m} \log \sum_{y \in \widehat{f}^{-m}\left(x_{0}\right)} \exp \left(\widehat{S}_{m}(\widehat{\varphi})(y)\right)>\widehat{\varphi}\left(x_{0}\right) .
$$

This is equivalent to show that the radius of convergence of the series

$$
\Xi(s):=\sum_{m=0}^{+\infty}\left(\sum_{z \in \widehat{f}^{-m}\left(x_{0}\right)} \exp \left(\widehat{S}_{m}(\widehat{\varphi})(z)\right)\right) s^{m}
$$

is strictly less than $\exp \left(-\widehat{\varphi}\left(x_{0}\right)\right)$. The proof of this fact is similar to Case 1 of the proof of [IRRL12, Proposition 4.1]. We include it here for completeness.

Put $\widehat{K}:=\bigcap_{i=0}^{+\infty} \widehat{f}^{-i}(U)$ and observe that $x_{0}$ is contained in this set. Consider the itinerary map

$$
\iota: \widehat{K} \rightarrow\{0,1\}^{\{1,2, \ldots\}}
$$

defined so that for every $i$ in $\{1,2, \ldots\}$ the point $\widehat{f}^{i}(z)$ is in $U_{\iota(z)_{i}}$. Since $\widehat{f}$ maps each of the sets $U_{0}$ and $U_{1}$ onto $f^{2 N k^{\prime}}\left(\overline{B\left(z^{\prime}, \varepsilon\right)}\right)$, and both of $U_{0}$ and $U_{1}$ are contained in this set, for every integer $k \geq 0$ and every sequence $a_{0}, a_{1}, \ldots, a_{k}$ of elements of $\{0,1\}$ there is a point of $\widehat{f}^{-(k+1)}\left(x_{0}\right)$ in the set $\widehat{K}\left(a_{0} a_{1} \cdots a_{k}\right):=\left\{z \in \widehat{K}\right.$ : for every $i$ in $\{0,1, \cdots, k\}$ we have $\left.\iota(z)_{i}=a_{i}\right\}$. By the Polynomial Shrinking of Components condition and our choice of $\phi$ and $U_{0}$, there is a constant $\widehat{C}>0$ such that for every integer $k \geq 1$ and every point $z$ in $\widehat{K}(\underbrace{0 \cdots 0}_{k})$, we have

$$
\widehat{S}_{k}(\widehat{\varphi})(z) \geq k \widehat{\varphi}\left(x_{0}\right)-\widehat{C} .
$$

Taking $\widehat{C}$ larger if necessary, assume that for every point $z$ in $U$ we have

$$
\widehat{\varphi}(z) \geq \widehat{\varphi}\left(x_{0}\right)-\widehat{C} \text {. }
$$

It follows that for every $k \geq 0$ and every sequence $a_{0}, a_{1}, \cdots, a_{k}$ of elements of $\{0,1\}$ with $a_{0}=1$ and every point $x$ in $\widehat{K}\left(a_{0} a_{1} \cdots a_{k}\right)$, we have

$$
\widehat{S}_{k+1}(\widehat{\varphi})(x) \geq(k+1) \widehat{\varphi}\left(x_{0}\right)-2\left(a_{0}+a_{1}+\cdots+a_{k}\right) \widehat{C} .
$$

In fact, put $\ell:=a_{0}+\cdots+a_{k}, i_{\ell+1}:=k+1$, and let

$$
0=i_{1}<i_{2}<\cdots<i_{\ell} \leq k
$$

be all integers $i$ in $\{0,1, \cdots, k\}$ such that $a_{i}=1$. Then by (4.5) and (4.6) for every $j \in\{1, \cdots, \ell\}$ we have

$$
\widehat{S}_{i_{j+1}-i_{j}}(\widehat{\varphi})\left(\widehat{f}^{i_{j}}(x)\right) \geq\left(i_{j+1}-i_{j}\right) \widehat{\varphi}\left(x_{0}\right)-2 \widehat{C} .
$$

Summing over $j$ in $\{1,2, \cdots, \ell\}$ we obtain (4.7). Thus, if we put

$$
\Phi(s):=\sum_{k=1}^{+\infty} \exp \left(k \widehat{\varphi}\left(x_{0}\right)-2 \widehat{C}\right) s^{k}
$$


then each of the coefficients of

$$
\Upsilon(s):=\Phi(s)+\Phi(s)^{2}+\cdots
$$

is less than or equal to the corresponding coefficient of $\Xi$, and therefore the radius of convergence of $\Xi$ is less than or equal to that of $\Upsilon$. Since clearly $\Phi(s) \rightarrow+\infty$ as $s \rightarrow \exp \left(-\widehat{\varphi}\left(x_{0}\right)\right)^{-}$, there is $s_{0}$ in $\left(0, \exp \left(-\widehat{\varphi}\left(x_{0}\right)\right)\right)$ such that $\Phi\left(s_{0}\right) \geq 1$. It follows that the radius of convergence of $\Upsilon$, and hence that of $\Xi$, is less than or equal to $s_{0}$ and therefore it is strictly less than $\exp \left(-\widehat{\varphi}\left(x_{0}\right)\right)$. This completes the proof of (4.4) and of the lemma.

Proof of the Key Lemma. When $\nu$ is supported on a periodic orbit, the desired inequality follows from Lemma 4.1 in the real case. In the complex case, note first that the hypotheses that $\beta>1$ and that $f$ satisfies the Polynomial Shrinking of Components condition with exponent $\beta$, imply that $f$ has no neutral periodic point in $J(f)$. So, in this case the Key Lemma follows from Case 1 in the proof of [IRRL12, Proposition 4.1].

Suppose $\nu$ is not supported on a periodic orbit. By Proposition 3.1 there is $D>0$, a connected and compact subset $B_{0}$ of $\operatorname{dom}(f)$ that intersects $J(f)$, and a free IMFS $\left(\phi_{k}\right)_{k=1}^{+\infty}$ generated by $f$ with time sequence $\left(m_{k}\right)_{k=1}^{+\infty}$ that is defined on $B_{0}$, and such that for every $k \geq 1$ and every point $y$ in $\phi_{k}\left(B_{0}\right)$ we have

$$
S_{m_{k}}(\varphi)(y) \geq m_{k} \int_{J(f)} \varphi d \nu-D
$$

Since the IMFS $\left(\phi_{k}\right)_{k=1}^{+\infty}$ is free, there is a point $x_{0}$ of $B_{0}$ in $J(f)$ such that for every $\underline{l}$ and $\underline{l}^{\prime}$ in $\Sigma^{*}$ such that $m_{\underline{l}}=m_{\underline{l}^{\prime}}$, the sets $\phi_{\underline{l}}\left(x_{0}\right)$ and $\phi_{\underline{l}^{\prime}}\left(x_{0}\right)$ are disjoint. Note that for every integer $k \geq 1$, every $\underline{l}=l_{1} \cdots l_{k}$ in $\Sigma^{*}$, every $y_{0}$ in $\phi_{\underline{l}}\left(x_{0}\right)$, and every $j$ in $\{1, \ldots, k-1\}$, the point

$$
y_{j}:=f^{m_{l_{1}}+m_{l_{2}}+\cdots+m_{l_{j}}}\left(y_{0}\right)
$$

is in $\phi_{m_{l_{j+1}}}\left(B_{0}\right)$. Therefore, by (4.8) we have

$$
\begin{aligned}
S_{m_{\underline{l}}}(\varphi)\left(y_{0}\right)=S_{m_{l_{1}}}(\varphi)\left(y_{0}\right)+S_{m_{l_{2}}}(\varphi)\left(y_{1}\right)+\cdots+S_{m_{l_{k}}}(\varphi)\left(y_{k-1}\right) \\
\quad \geq \sum_{i=1}^{k}\left(m_{l_{i}} \int_{J(f)} \varphi d \nu-D\right)=m_{\underline{l}} \int_{J(f)} \varphi d \nu-k D .
\end{aligned}
$$

This shows that for every $\underline{l}$ in $\Sigma^{*}$, and every $y_{0}$ in $\phi_{\underline{l}}\left(x_{0}\right)$ we have

$$
\exp \left(S_{m_{\underline{l}}}(\varphi)\left(y_{0}\right)\right) \geq \exp \left(m_{\underline{l}} \int_{J(f)} \varphi d \nu\right) \exp (-|\underline{l}| D) .
$$

On the other hand, if for every integer $n \geq 1$ we put

$$
\Xi_{n}:=\bigcup_{\underline{l} \in \Sigma^{*}, m_{\underline{l}}=n} \phi_{\underline{l}}\left(x_{0}\right)
$$


then the radius of convergence of the series

$$
\Xi(s):=\sum_{n=1}^{+\infty}\left(\sum_{y \in \Xi_{n}} \exp \left(S_{n}(\varphi)(y)\right)\right) s^{n}
$$

is given by

$$
R:=\left(\limsup _{n \rightarrow+\infty}\left(\sum_{y \in \Xi_{n}} \exp \left(S_{n}(\varphi)(y)\right)\right)^{1 / n}\right)^{-1}
$$

and satisfies

$$
\exp \left(-\limsup _{n \rightarrow+\infty} \frac{1}{n} \log \sum_{y \in f^{-n}\left(x_{0}\right)} \exp \left(S_{n}(\varphi)(y)\right)\right) \leq R .
$$

Therefore, to complete the proof of the Key Lemma it suffices to prove $R<$ $\exp \left(-\int_{J(f)} \varphi d \nu\right)$. Put

$$
\Phi(s):=\sum_{l=1}^{+\infty} \exp (-D) \exp \left(m_{l} \int_{J(f)} \varphi d \nu\right) s^{m_{l}} .
$$

By inequality (4.9) and the fact that $\left(\phi_{k}\right)_{k=1}^{+\infty}$ is free, each of the coefficients of the series

$$
\Upsilon(s):=\sum_{i=1}^{+\infty} \Phi(s)^{i}=\sum_{n=1}^{+\infty}\left(\sum_{\underline{\underline{l}} \in \Sigma^{*}, m_{\underline{l}}=n} \exp \left(m_{\underline{l}} \int_{J(f)} \varphi d \nu\right) \exp (-|\underline{l}| D)\right) s^{n},
$$

does not exceed the corresponding coefficient of the series $\Xi$, so the radius of convergence of $\Xi$ is less than or equal to that of $\Upsilon$. Since clearly $\Phi(s) \rightarrow+\infty$ as $s \rightarrow \exp \left(-\int_{J(f)} \varphi d \nu\right)^{-}$, there exists $s_{0}$ in $\left(0, \exp \left(-\int_{J(f)} \varphi d \nu\right)\right)$ such that $\Phi\left(s_{0}\right) \geq 1$. This implies that the radius of convergence of $\Upsilon$, and hence that of $\Xi$, is less than or equal to $s_{0}$, and therefore that $R \leq s_{0}<$ $\exp \left(-\int_{J(f)} \varphi d \nu\right)$. This completes the proof of the Key Lemma.

\section{REFERENCES}

[Bal00] Viviane Baladi. Positive transfer operators and decay of correlations, volume 16 of Advanced Series in Nonlinear Dynamics. World Scientific Publishing Co. Inc., River Edge, NJ, 2000.

[BK90] V. Baladi and G. Keller. Zeta functions and transfer operators for piecewise monotone transformations. Comm. Math. Phys., 127(3):459-477, 1990.

[Bow75] Rufus Bowen. Equilibrium states and the ergodic theory of Anosov diffeomorphisms. Lecture Notes in Mathematics, Vol. 470. Springer-Verlag, Berlin, 1975.

[BPS01] Jérôme Buzzi, Frédéric Paccaut, and Bernard Schmitt. Conformal measures for multidimensional piecewise invertible maps. Ergodic Theory Dynam. Systems, 21(4):1035-1049, 2001. 
[BRLSvS08] H. Bruin, J. Rivera-Letelier, W. Shen, and S. van Strien. Large derivatives, backward contraction and invariant densities for interval maps. Invent. Math., 172(3):509-533, 2008.

[BT08] Henk Bruin and Mike Todd. Equilibrium states for interval maps: potentials with $\sup \phi-\inf \phi<h_{\text {top }}(f)$. Comm. Math. Phys., 283(3):579-611, 2008.

[BT11] Henk Bruin and Mike Todd. Erratum to: Equilibrium states for interval maps: potentials with $\sup \phi-\inf \phi<h_{\text {top }}(f)$. Comm. Math. Phys., 304(2):583-584, 2011.

[Buz04] Jérôme Buzzi. Entropy of equilibrium measures of continuous piecewise monotonic maps. Stoch. Dyn., 4(1):84-94, 2004.

[CG93] Lennart Carleson and Theodore W. Gamelin. Complex dynamics. Universitext: Tracts in Mathematics. Springer-Verlag, New York, 1993.

[DKU90] Manfred Denker, Gerhard Keller, and Mariusz Urbański. On the uniqueness of equilibrium states for piecewise monotone mappings. Studia Math., 97(1):27-36, 1990.

[dMvS93] Welington de Melo and Sebastian van Strien. One-dimensional dynamics, volume 25 of Ergebnisse der Mathematik und ihrer Grenzgebiete (3) [Results in Mathematics and Related Areas (3)]. Springer-Verlag, Berlin, 1993.

[DPU96] Manfred Denker, Feliks Przytycki, and Mariusz Urbański. On the transfer operator for rational functions on the Riemann sphere. Ergodic Theory Dynam. Systems, 16(2):255-266, 1996.

[DU91] M. Denker and M. Urbański. Ergodic theory of equilibrium states for rational maps. Nonlinearity, 4(1):103-134, 1991.

[FLM83] Alexandre Freire, Artur Lopes, and Ricardo Mañé. An invariant measure for rational maps. Bol. Soc. Brasil. Mat., 14(1):45-62, 1983.

[FU00] Albert M. Fisher and Mariusz Urbański. On invariant line fields. Bull. London Math. Soc., 32(5):555-570, 2000.

[Gou05] Sébastien Gouëzel. Berry-Esseen theorem and local limit theorem for non uniformly expanding maps. Ann. Inst. H. Poincaré Probab. Statist., 41(6):9971024, 2005.

[Hay99] Nicolai Haydn. Convergence of the transfer operator for rational maps. Ergodic Theory Dynam. Systems, 19(3):657-669, 1999.

[Hay00] Nicolai Haydn. Statistical properties of equilibrium states for rational maps. Ergodic Theory Dynam. Systems, 20(5):1371-1390, 2000.

[HK82] Franz Hofbauer and Gerhard Keller. Ergodic properties of invariant measures for piecewise monotonic transformations. Math. Z., 180(1):119-140, 1982.

[IRRL12] Irene Inoquio-Renteria and Juan Rivera-Letelier. A characterization of hyperbolic potentials of rational maps. Bull. Braz. Math. Soc. (N.S.), 43(1):99127,2012

[Kel85] Gerhard Keller. Generalized bounded variation and applications to piecewise monotonic transformations. Z. Wahrsch. Verw. Gebiete, 69(3):461-478, 1985.

[KN95] Gerhard Keller and Tomasz Nowicki. Fibonacci maps re(al)visited. Ergodic Theory Dynam. Systems, 15(1):99-120, 1995.

[KvS09] Oleg Kozlovski and Sebastian van Strien. Local connectivity and quasiconformal rigidity of non-renormalizable polynomials. Proc. Lond. Math. Soc. (3), 99(2):275-296, 2009.

[Li13] Huaibin Li. Large deviation principles of one-dimensional maps for Hölder continuous potentials. arXiv:1307.6433v1, 2013.

[Lju83] M. Ju. Ljubich. Entropy properties of rational endomorphisms of the Riemann sphere. Ergodic Theory Dynam. Systems, 3(3):351-385, 1983.

[LM93] Mikhail Lyubich and John Milnor. The Fibonacci unimodal map. J. Amer. Math. Soc., 6(2):425-457, 1993. 
[Lop93] A. O. Lopes. The zeta function, nondifferentiability of pressure, and the critical exponent of transition. Adv. Math., 101(2):133-165, 1993.

[LRL13] Huaibin Li and Juan Rivera-Letelier. Equilibrium states of interval maps for hyperbolic potentials. arXiv:1210.6952 v2, 2013.

[LS10] HuaiBin Li and WeiXiao Shen. On non-uniform hyperbolicity assumptions in one-dimensional dynamics. Sci. China Math., 53(7):1663-1677, 2010.

[LSV98] Carlangelo Liverani, Benoit Saussol, and Sandro Vaienti. Conformal measure and decay of correlation for covering weighted systems. Ergodic Theory Dynam. Systems, 18(6):1399-1420, 1998.

[LSV99] Carlangelo Liverani, Benoît Saussol, and Sandro Vaienti. A probabilistic approach to intermittency. Ergodic Theory Dynam. Systems, 19(3):671-685, 1999.

[LY73] A. Lasota and James A. Yorke. On the existence of invariant measures for piecewise monotonic transformations. Trans. Amer. Math. Soc., 186:481-488 (1974), 1973.

[Mañ83] Ricardo Mañé. On the uniqueness of the maximizing measure for rational maps. Bol. Soc. Brasil. Mat., 14(1):27-43, 1983.

[Mil06] John Milnor. Dynamics in one complex variable, volume 160 of Annals of Mathematics Studies. Princeton University Press, Princeton, NJ, third edition, 2006.

[MN05] Ian Melbourne and Matthew Nicol. Almost sure invariance principle for nonuniformly hyperbolic systems. Comm. Math. Phys., 260(1):131-146, 2005.

[MN08] Ian Melbourne and Matthew Nicol. Large deviations for nonuniformly hyperbolic systems. Trans. Amer. Math. Soc., 360(12):6661-6676, 2008.

[MT12] Ian Melbourne and Dalia Terhesiu. Operator renewal theory and mixing rates for dynamical systems with infinite measure. Invent. Math., 189(1):61-110, 2012.

[PM80] Yves Pomeau and Paul Manneville. Intermittent transition to turbulence in dissipative dynamical systems. Comm. Math. Phys., 74(2):189-197, 1980.

[PRL11] Feliks Przytycki and Juan Rivera-Letelier. Nice inducing schemes and the thermodynamics of rational maps. Comm. Math. Phys., 301(3):661-707, 2011.

[PRL13] Feliks Przytycki and Juan Rivera-Letelier. Geometric pressure for multimodal maps of the interval. 2013. Preliminary version available at http://www.impan.pl/ feliksp/interval24a.pdf.

[PRLS03] Feliks Przytycki, Juan Rivera-Letelier, and Stanislav Smirnov. Equivalence and topological invariance of conditions for non-uniform hyperbolicity in the iteration of rational maps. Invent. Math., 151(1):29-63, 2003.

[PRLS04] Feliks Przytycki, Juan Rivera-Letelier, and Stanislav Smirnov. Equality of pressures for rational functions. Ergodic Theory Dynam. Systems, 24(3):891914, 2004.

[Prz90] Feliks Przytycki. On the Perron-Frobenius-Ruelle operator for rational maps on the Riemann sphere and for Hölder continuous functions. Bol. Soc. Brasil. Mat. (N.S.), 20(2):95-125, 1990.

[PS92] Thomas Prellberg and Joseph Slawny. Maps of intervals with indifferent fixed points: thermodynamic formalism and phase transitions. J. Statist. Phys., 66(1-2):503-514, 1992.

[PSY98] Mark Pollicott, Richard Sharp, and Michiko Yuri. Large deviations for maps with indifferent fixed points. Nonlinearity, 11(4):1173-1184, 1998.

[PU10] Feliks Przytycki and Mariusz Urbański. Conformal fractals: ergodic theory methods, volume 371 of London Mathematical Society Lecture Note Series. Cambridge University Press, Cambridge, 2010. 
[PW99] Mark Pollicott and Howard Weiss. Multifractal analysis of Lyapunov exponent for continued fraction and Manneville-Pomeau transformations and applications to Diophantine approximation. Comm. Math. Phys., 207(1):145$171,1999$.

[RL07] Juan Rivera-Letelier. A connecting lemma for rational maps satisfying a nogrowth condition. Ergodic Theory Dynam. Systems, 27(2):595-636, 2007.

[RL12a] Juan Rivera-Letelier. Asymptotic expansion of smooth interval maps. 2012. arXiv:1204.3071 2 .

[RL12b] Juan Rivera-Letelier. On the asymptotic expansion of maps with disconnected Julia set. 2012. arXiv:1206.2376v1.

[RLS10] Juan Rivera-Letelier and Weixiao Shen. Statistical properties of one-dimensional maps under weak hyperbolicity assumptions. 2010. arXiv:1004.0230v1.

[Rue76] David Ruelle. A measure associated with axiom-A attractors. Amer. J. Math., 98(3):619-654, 1976.

[Rue94] David Ruelle. Dynamical zeta functions for piecewise monotone maps of the interval, volume 4 of CRM Monograph Series. American Mathematical Society, Providence, RI, 1994.

[Sar01] Omri M. Sarig. Phase transitions for countable Markov shifts. Comm. Math. Phys., 217(3):555-577, 2001.

[Sin72] Ja. G. Sină̌. Gibbs measures in ergodic theory. Uspehi Mat. Nauk, 27(4(166)):21-64, 1972.

[SUZ11] M. Szostakiewicz, M. Urbański, and A. Zdunik. Fine inducing and equilibrium measures for fational functions of the Riemann sphere. Available at: http://www . math. unt .edu/ urbanski/papers/FI-1-Dim20110924.pdf, 2011.

[TK05] Marta Tyran-Kamińska. An invariance principle for maps with polynomial decay of correlations. Comm. Math. Phys., 260(1):1-15, 2005.

[Wal78] Peter Walters. Equilibrium states for $\beta$-transformations and related transformations. Math. Z., 159(1):65-88, 1978.

[You99] Lai-Sang Young. Recurrence times and rates of mixing. Israel J. Math., 110:153-188, 1999.

\section{REFERENCES}

[Bal00] Viviane Baladi. Positive transfer operators and decay of correlations, volume 16 of Advanced Series in Nonlinear Dynamics. World Scientific Publishing Co. Inc., River Edge, NJ, 2000.

[BK90] V. Baladi and G. Keller. Zeta functions and transfer operators for piecewise monotone transformations. Comm. Math. Phys., 127(3):459-477, 1990.

[Bow75] Rufus Bowen. Equilibrium states and the ergodic theory of Anosov diffeomorphisms. Lecture Notes in Mathematics, Vol. 470. Springer-Verlag, Berlin, 1975 .

[BPS01] Jérôme Buzzi, Frédéric Paccaut, and Bernard Schmitt. Conformal measures for multidimensional piecewise invertible maps. Ergodic Theory Dynam. Systems, 21(4):1035-1049, 2001.

[BRLSvS08] H. Bruin, J. Rivera-Letelier, W. Shen, and S. van Strien. Large derivatives, backward contraction and invariant densities for interval maps. Invent. Math., 172(3):509-533, 2008.

[BT08] Henk Bruin and Mike Todd. Equilibrium states for interval maps: potentials with $\sup \phi-\inf \phi<h_{\text {top }}(f)$. Comm. Math. Phys., 283(3):579-611, 2008. 
[BT11] Henk Bruin and Mike Todd. Erratum to: Equilibrium states for interval maps: potentials with $\sup \phi-\inf \phi<h_{\text {top }}(f)$. Comm. Math. Phys., 304(2):583-584, 2011.

[Buz04] Jérôme Buzzi. Entropy of equilibrium measures of continuous piecewise monotonic maps. Stoch. Dyn., 4(1):84-94, 2004.

[CG93] Lennart Carleson and Theodore W. Gamelin. Complex dynamics. Universitext: Tracts in Mathematics. Springer-Verlag, New York, 1993.

[DKU90] Manfred Denker, Gerhard Keller, and Mariusz Urbański. On the uniqueness of equilibrium states for piecewise monotone mappings. Studia Math., 97(1):27-36, 1990.

[dMvS93] Welington de Melo and Sebastian van Strien. One-dimensional dynamics, volume 25 of Ergebnisse der Mathematik und ihrer Grenzgebiete (3) [Results in Mathematics and Related Areas (3)]. Springer-Verlag, Berlin, 1993.

[DPU96] Manfred Denker, Feliks Przytycki, and Mariusz Urbański. On the transfer operator for rational functions on the Riemann sphere. Ergodic Theory Dynam. Systems, 16(2):255-266, 1996.

[DU91] M. Denker and M. Urbański. Ergodic theory of equilibrium states for rational maps. Nonlinearity, 4(1):103-134, 1991.

[FLM83] Alexandre Freire, Artur Lopes, and Ricardo Mañé. An invariant measure for rational maps. Bol. Soc. Brasil. Mat., 14(1):45-62, 1983.

[FU00] Albert M. Fisher and Mariusz Urbański. On invariant line fields. Bull. London Math. Soc., 32(5):555-570, 2000.

[Gou05] Sébastien Gouëzel. Berry-Esseen theorem and local limit theorem for non uniformly expanding maps. Ann. Inst. H. Poincaré Probab. Statist., 41(6):9971024, 2005.

[Hay99] Nicolai Haydn. Convergence of the transfer operator for rational maps. Ergodic Theory Dynam. Systems, 19(3):657-669, 1999.

[Hay00] Nicolai Haydn. Statistical properties of equilibrium states for rational maps. Ergodic Theory Dynam. Systems, 20(5):1371-1390, 2000.

[HK82] Franz Hofbauer and Gerhard Keller. Ergodic properties of invariant measures for piecewise monotonic transformations. Math. Z., 180(1):119-140, 1982.

[IRRL12] Irene Inoquio-Renteria and Juan Rivera-Letelier. A characterization of hyperbolic potentials of rational maps. Bull. Braz. Math. Soc. (N.S.), 43(1):99$127,2012$.

[Kel85] Gerhard Keller. Generalized bounded variation and applications to piecewise monotonic transformations. Z. Wahrsch. Verw. Gebiete, 69(3):461-478, 1985.

[KN95] Gerhard Keller and Tomasz Nowicki. Fibonacci maps re(al)visited. Ergodic Theory Dynam. Systems, 15(1):99-120, 1995.

[KvS09] Oleg Kozlovski and Sebastian van Strien. Local connectivity and quasiconformal rigidity of non-renormalizable polynomials. Proc. Lond. Math. Soc. (3), 99(2):275-296, 2009.

[Li13] Huaibin Li. Large deviation principles of one-dimensional maps for Hölder continuous potentials. arXiv:1307.6433v1, 2013.

[Lju83] M. Ju. Ljubich. Entropy properties of rational endomorphisms of the Riemann sphere. Ergodic Theory Dynam. Systems, 3(3):351-385, 1983.

[LM93] Mikhail Lyubich and John Milnor. The Fibonacci unimodal map. J. Amer. Math. Soc., 6(2):425-457, 1993.

[Lop93] A. O. Lopes. The zeta function, nondifferentiability of pressure, and the critical exponent of transition. Adv. Math., 101(2):133-165, 1993.

[LRL13] Huaibin Li and Juan Rivera-Letelier. Equilibrium states of interval maps for hyperbolic potentials. arXiv:1210.6952 v2, 2013.

[LS10] HuaiBin Li and WeiXiao Shen. On non-uniform hyperbolicity assumptions in one-dimensional dynamics. Sci. China Math., 53(7):1663-1677, 2010. 
[LSV98] Carlangelo Liverani, Benoit Saussol, and Sandro Vaienti. Conformal measure and decay of correlation for covering weighted systems. Ergodic Theory Dynam. Systems, 18(6):1399-1420, 1998.

[LSV99] Carlangelo Liverani, Benoît Saussol, and Sandro Vaienti. A probabilistic approach to intermittency. Ergodic Theory Dynam. Systems, 19(3):671-685, 1999.

[LY73] A. Lasota and James A. Yorke. On the existence of invariant measures for piecewise monotonic transformations. Trans. Amer. Math. Soc., 186:481-488 (1974), 1973.

[Mañ83] Ricardo Mañé. On the uniqueness of the maximizing measure for rational maps. Bol. Soc. Brasil. Mat., 14(1):27-43, 1983.

[Mil06] John Milnor. Dynamics in one complex variable, volume 160 of Annals of Mathematics Studies. Princeton University Press, Princeton, NJ, third edition, 2006.

[MN05] Ian Melbourne and Matthew Nicol. Almost sure invariance principle for nonuniformly hyperbolic systems. Comm. Math. Phys., 260(1):131-146, 2005.

[MN08] Ian Melbourne and Matthew Nicol. Large deviations for nonuniformly hyperbolic systems. Trans. Amer. Math. Soc., 360(12):6661-6676, 2008.

[MT12] Ian Melbourne and Dalia Terhesiu. Operator renewal theory and mixing rates for dynamical systems with infinite measure. Invent. Math., 189(1):61-110, 2012.

[PM80] Yves Pomeau and Paul Manneville. Intermittent transition to turbulence in dissipative dynamical systems. Comm. Math. Phys., 74(2):189-197, 1980.

[PRL11] Feliks Przytycki and Juan Rivera-Letelier. Nice inducing schemes and the thermodynamics of rational maps. Comm. Math. Phys., 301(3):661-707, 2011.

[PRL13] Feliks Przytycki and Juan Rivera-Letelier. Geometric pressure for multimodal maps of the interval. 2013. Preliminary version available at http://www.impan.pl/ feliksp/interval24a.pdf.

[PRLS03] Feliks Przytycki, Juan Rivera-Letelier, and Stanislav Smirnov. Equivalence and topological invariance of conditions for non-uniform hyperbolicity in the iteration of rational maps. Invent. Math., 151(1):29-63, 2003.

[PRLS04] Feliks Przytycki, Juan Rivera-Letelier, and Stanislav Smirnov. Equality of pressures for rational functions. Ergodic Theory Dynam. Systems, 24(3):891914,2004

[Prz90] Feliks Przytycki. On the Perron-Frobenius-Ruelle operator for rational maps on the Riemann sphere and for Hölder continuous functions. Bol. Soc. Brasil. Mat. (N.S.), 20(2):95-125, 1990.

[PS92] Thomas Prellberg and Joseph Slawny. Maps of intervals with indifferent fixed points: thermodynamic formalism and phase transitions. J. Statist. Phys., 66(1-2):503-514, 1992.

[PSY98] Mark Pollicott, Richard Sharp, and Michiko Yuri. Large deviations for maps with indifferent fixed points. Nonlinearity, 11(4):1173-1184, 1998.

[PU10] Feliks Przytycki and Mariusz Urbański. Conformal fractals: ergodic theory methods, volume 371 of London Mathematical Society Lecture Note Series. Cambridge University Press, Cambridge, 2010.

[PW99] Mark Pollicott and Howard Weiss. Multifractal analysis of Lyapunov exponent for continued fraction and Manneville-Pomeau transformations and applications to Diophantine approximation. Comm. Math. Phys., 207(1):145$171,1999$.

[RL07] Juan Rivera-Letelier. A connecting lemma for rational maps satisfying a nogrowth condition. Ergodic Theory Dynam. Systems, 27(2):595-636, 2007. 
[RL12a] Juan Rivera-Letelier. Asymptotic expansion of smooth interval maps. 2012. arXiv:1204.3071 2 .

[RL12b] Juan Rivera-Letelier. On the asymptotic expansion of maps with disconnected Julia set. 2012. arXiv:1206.2376 1 .

[RLS10] Juan Rivera-Letelier and Weixiao Shen. Statistical properties of one-dimensional maps under weak hyperbolicity assumptions. 2010. arXiv:1004.0230v1.

[Rue76] David Ruelle. A measure associated with axiom-A attractors. Amer. J. Math., 98(3):619-654, 1976.

[Rue94] David Ruelle. Dynamical zeta functions for piecewise monotone maps of the interval, volume 4 of CRM Monograph Series. American Mathematical Society, Providence, RI, 1994.

[Sar01] Omri M. Sarig. Phase transitions for countable Markov shifts. Comm. Math. Phys., 217(3):555-577, 2001.

[Sin72] Ja. G. Sinal̆. Gibbs measures in ergodic theory. Uspehi Mat. Nauk, 27(4(166)):21-64, 1972 .

[SUZ11] M. Szostakiewicz, M. Urbański, and A. Zdunik. Fine inducing and equilibrium measures for fational functions of the Riemann sphere. Available at: http://www . math. unt . edu/ urbanski/papers/FI-1-Dim20110924.pdf, 2011.

[TK05] Marta Tyran-Kamińska. An invariance principle for maps with polynomial decay of correlations. Comm. Math. Phys., 260(1):1-15, 2005.

[Wal78] Peter Walters. Equilibrium states for $\beta$-transformations and related transformations. Math. Z., 159(1):65-88, 1978.

[You99] Lai-Sang Young. Recurrence times and rates of mixing. Israel J. Math., 110:153-188, 1999.

\section{REFERENCES}

[Bal00] Viviane Baladi. Positive transfer operators and decay of correlations, volume 16 of Advanced Series in Nonlinear Dynamics. World Scientific Publishing Co. Inc., River Edge, NJ, 2000.

[BK90] V. Baladi and G. Keller. Zeta functions and transfer operators for piecewise monotone transformations. Comm. Math. Phys., 127(3):459-477, 1990.

[Bow75] Rufus Bowen. Equilibrium states and the ergodic theory of Anosov diffeomorphisms. Lecture Notes in Mathematics, Vol. 470. Springer-Verlag, Berlin, 1975.

[BPS01] Jérôme Buzzi, Frédéric Paccaut, and Bernard Schmitt. Conformal measures for multidimensional piecewise invertible maps. Ergodic Theory Dynam. Systems, 21(4):1035-1049, 2001.

[BRLSvS08] H. Bruin, J. Rivera-Letelier, W. Shen, and S. van Strien. Large derivatives, backward contraction and invariant densities for interval maps. Invent. Math., 172(3):509-533, 2008.

[BT08] Henk Bruin and Mike Todd. Equilibrium states for interval maps: potentials with $\sup \phi-\inf \phi<h_{\text {top }}(f)$. Comm. Math. Phys., 283(3):579-611, 2008.

[BT11] Henk Bruin and Mike Todd. Erratum to: Equilibrium states for interval maps: potentials with $\sup \phi-\inf \phi<h_{\text {top }}(f)$. Comm. Math. Phys., 304(2):583-584, 2011.

[Buz04] Jérôme Buzzi. Entropy of equilibrium measures of continuous piecewise monotonic maps. Stoch. Dyn., 4(1):84-94, 2004.

[CG93] Lennart Carleson and Theodore W. Gamelin. Complex dynamics. Universitext: Tracts in Mathematics. Springer-Verlag, New York, 1993. 
[DKU90] Manfred Denker, Gerhard Keller, and Mariusz Urbański. On the uniqueness of equilibrium states for piecewise monotone mappings. Studia Math., 97(1):27-36, 1990.

[dMvS93] Welington de Melo and Sebastian van Strien. One-dimensional dynamics, volume 25 of Ergebnisse der Mathematik und ihrer Grenzgebiete (3) [Results in Mathematics and Related Areas (3)]. Springer-Verlag, Berlin, 1993.

[DPU96] Manfred Denker, Feliks Przytycki, and Mariusz Urbański. On the transfer operator for rational functions on the Riemann sphere. Ergodic Theory Dynam. Systems, 16(2):255-266, 1996.

[DU91] M. Denker and M. Urbański. Ergodic theory of equilibrium states for rational maps. Nonlinearity, 4(1):103-134, 1991.

[FLM83] Alexandre Freire, Artur Lopes, and Ricardo Mañé. An invariant measure for rational maps. Bol. Soc. Brasil. Mat., 14(1):45-62, 1983.

[FU00] Albert M. Fisher and Mariusz Urbański. On invariant line fields. Bull. London Math. Soc., 32(5):555-570, 2000.

[Gou05] Sébastien Gouëzel. Berry-Esseen theorem and local limit theorem for non uniformly expanding maps. Ann. Inst. H. Poincaré Probab. Statist., 41(6):9971024, 2005.

[Hay99] Nicolai Haydn. Convergence of the transfer operator for rational maps. Ergodic Theory Dynam. Systems, 19(3):657-669, 1999.

[Hay00] Nicolai Haydn. Statistical properties of equilibrium states for rational maps. Ergodic Theory Dynam. Systems, 20(5):1371-1390, 2000.

[HK82] Franz Hofbauer and Gerhard Keller. Ergodic properties of invariant measures for piecewise monotonic transformations. Math. Z., 180(1):119-140, 1982.

[IRRL12] Irene Inoquio-Renteria and Juan Rivera-Letelier. A characterization of hyperbolic potentials of rational maps. Bull. Braz. Math. Soc. (N.S.), 43(1):99$127,2012$.

[Kel85] Gerhard Keller. Generalized bounded variation and applications to piecewise monotonic transformations. Z. Wahrsch. Verw. Gebiete, 69(3):461-478, 1985.

[KN95] Gerhard Keller and Tomasz Nowicki. Fibonacci maps re(al)visited. Ergodic Theory Dynam. Systems, 15(1):99-120, 1995.

[KvS09] Oleg Kozlovski and Sebastian van Strien. Local connectivity and quasiconformal rigidity of non-renormalizable polynomials. Proc. Lond. Math. Soc. (3), 99(2):275-296, 2009.

[Li13] Huaibin Li. Large deviation principles of one-dimensional maps for Hölder continuous potentials. arXiv:1307.6433v1, 2013.

[Lju83] M. Ju. Ljubich. Entropy properties of rational endomorphisms of the Riemann sphere. Ergodic Theory Dynam. Systems, 3(3):351-385, 1983.

[LM93] Mikhail Lyubich and John Milnor. The Fibonacci unimodal map. J. Amer. Math. Soc., 6(2):425-457, 1993.

[Lop93] A. O. Lopes. The zeta function, nondifferentiability of pressure, and the critical exponent of transition. Adv. Math., 101(2):133-165, 1993.

[LRL13] Huaibin Li and Juan Rivera-Letelier. Equilibrium states of interval maps for hyperbolic potentials. arXiv:1210.6952 v2, 2013.

[LS10] HuaiBin Li and WeiXiao Shen. On non-uniform hyperbolicity assumptions in one-dimensional dynamics. Sci. China Math., 53(7):1663-1677, 2010.

[LSV98] Carlangelo Liverani, Benoit Saussol, and Sandro Vaienti. Conformal measure and decay of correlation for covering weighted systems. Ergodic Theory Dynam. Systems, 18(6):1399-1420, 1998.

[LSV99] Carlangelo Liverani, Benoît Saussol, and Sandro Vaienti. A probabilistic approach to intermittency. Ergodic Theory Dynam. Systems, 19(3):671-685, 1999. 
[LY73] A. Lasota and James A. Yorke. On the existence of invariant measures for piecewise monotonic transformations. Trans. Amer. Math. Soc., 186:481-488 (1974), 1973.

[Mañ83] Ricardo Mañé. On the uniqueness of the maximizing measure for rational maps. Bol. Soc. Brasil. Mat., 14(1):27-43, 1983.

[Mil06] John Milnor. Dynamics in one complex variable, volume 160 of Annals of Mathematics Studies. Princeton University Press, Princeton, NJ, third edition, 2006.

[MN05] Ian Melbourne and Matthew Nicol. Almost sure invariance principle for nonuniformly hyperbolic systems. Comm. Math. Phys., 260(1):131-146, 2005.

[MN08] Ian Melbourne and Matthew Nicol. Large deviations for nonuniformly hyperbolic systems. Trans. Amer. Math. Soc., 360(12):6661-6676, 2008.

[MT12] Ian Melbourne and Dalia Terhesiu. Operator renewal theory and mixing rates for dynamical systems with infinite measure. Invent. Math., 189(1):61-110, 2012.

[PM80] Yves Pomeau and Paul Manneville. Intermittent transition to turbulence in dissipative dynamical systems. Comm. Math. Phys., 74(2):189-197, 1980.

[PRL11] Feliks Przytycki and Juan Rivera-Letelier. Nice inducing schemes and the thermodynamics of rational maps. Comm. Math. Phys., 301(3):661-707, 2011.

[PRL13] Feliks Przytycki and Juan Rivera-Letelier. Geometric pressure for multimodal maps of the interval. 2013. Preliminary version available at http://www.impan.pl/ feliksp/interval24a.pdf.

[PRLS03] Feliks Przytycki, Juan Rivera-Letelier, and Stanislav Smirnov. Equivalence and topological invariance of conditions for non-uniform hyperbolicity in the iteration of rational maps. Invent. Math., 151(1):29-63, 2003.

[PRLS04] Feliks Przytycki, Juan Rivera-Letelier, and Stanislav Smirnov. Equality of pressures for rational functions. Ergodic Theory Dynam. Systems, 24(3):891914, 2004.

[Prz90] Feliks Przytycki. On the Perron-Frobenius-Ruelle operator for rational maps on the Riemann sphere and for Hölder continuous functions. Bol. Soc. Brasil. Mat. (N.S.), 20(2):95-125, 1990.

[PS92] Thomas Prellberg and Joseph Slawny. Maps of intervals with indifferent fixed points: thermodynamic formalism and phase transitions. J. Statist. Phys., 66(1-2):503-514, 1992.

[PSY98] Mark Pollicott, Richard Sharp, and Michiko Yuri. Large deviations for maps with indifferent fixed points. Nonlinearity, 11(4):1173-1184, 1998.

[PU10] Feliks Przytycki and Mariusz Urbański. Conformal fractals: ergodic theory methods, volume 371 of London Mathematical Society Lecture Note Series. Cambridge University Press, Cambridge, 2010.

[PW99] Mark Pollicott and Howard Weiss. Multifractal analysis of Lyapunov exponent for continued fraction and Manneville-Pomeau transformations and applications to Diophantine approximation. Comm. Math. Phys., 207(1):145171, 1999.

[RL07] Juan Rivera-Letelier. A connecting lemma for rational maps satisfying a nogrowth condition. Ergodic Theory Dynam. Systems, 27(2):595-636, 2007.

[RL12a] Juan Rivera-Letelier. Asymptotic expansion of smooth interval maps. 2012. arXiv:1204.3071 v2.

[RL12b] Juan Rivera-Letelier. On the asymptotic expansion of maps with disconnected Julia set. 2012. arXiv:1206.2376v1. 
[RLS10] Juan Rivera-Letelier and Weixiao Shen. Statistical properties of one-dimensional maps under weak hyperbolicity assumptions. 2010. arXiv:1004.0230v1.

[Rue76] David Ruelle. A measure associated with axiom-A attractors. Amer. J. Math., 98(3):619-654, 1976.

[Rue94] David Ruelle. Dynamical zeta functions for piecewise monotone maps of the interval, volume 4 of CRM Monograph Series. American Mathematical Society, Providence, RI, 1994.

[Sar01] Omri M. Sarig. Phase transitions for countable Markov shifts. Comm. Math. Phys., 217(3):555-577, 2001.

[Sin72] Ja. G. Sinal. Gibbs measures in ergodic theory. Uspehi Mat. Nauk, 27(4(166)):21-64, 1972 .

[SUZ11] M. Szostakiewicz, M. Urbański, and A. Zdunik. Fine inducing and equilibrium measures for fational functions of the Riemann sphere. Available at: http://www . math.unt.edu/ urbanski/papers/FI-1-Dim20110924.pdf, 2011.

[TK05] Marta Tyran-Kamińska. An invariance principle for maps with polynomial decay of correlations. Comm. Math. Phys., 260(1):1-15, 2005.

[Wal78] Peter Walters. Equilibrium states for $\beta$-transformations and related transformations. Math. Z., 159(1):65-88, 1978.

[You99] Lai-Sang Young. Recurrence times and rates of mixing. Israel J. Math., 110:153-188, 1999.

Huaibin Li, Facultad de Matemáticas, Pontificia Universidad Católica de Chile, Avenida Vicuña Mackenna 4860, Santiago, Chile

E-mail address: matlihb@gmail.com

Juan Rivera-Letelier, Facultad de Matemáticas, Pontificia Universidad Católica de Chile, Avenida Vicuña Mackenna 4860, Santiago, Chile

E-mail address: riveraletelier@mat.puc.cl 